\title{
Zones of Restricted Activity for Protection of Key Fish Areas Along the Trans-Alaska Pipeline System on Federally-Administered Lands
}

Second Edition

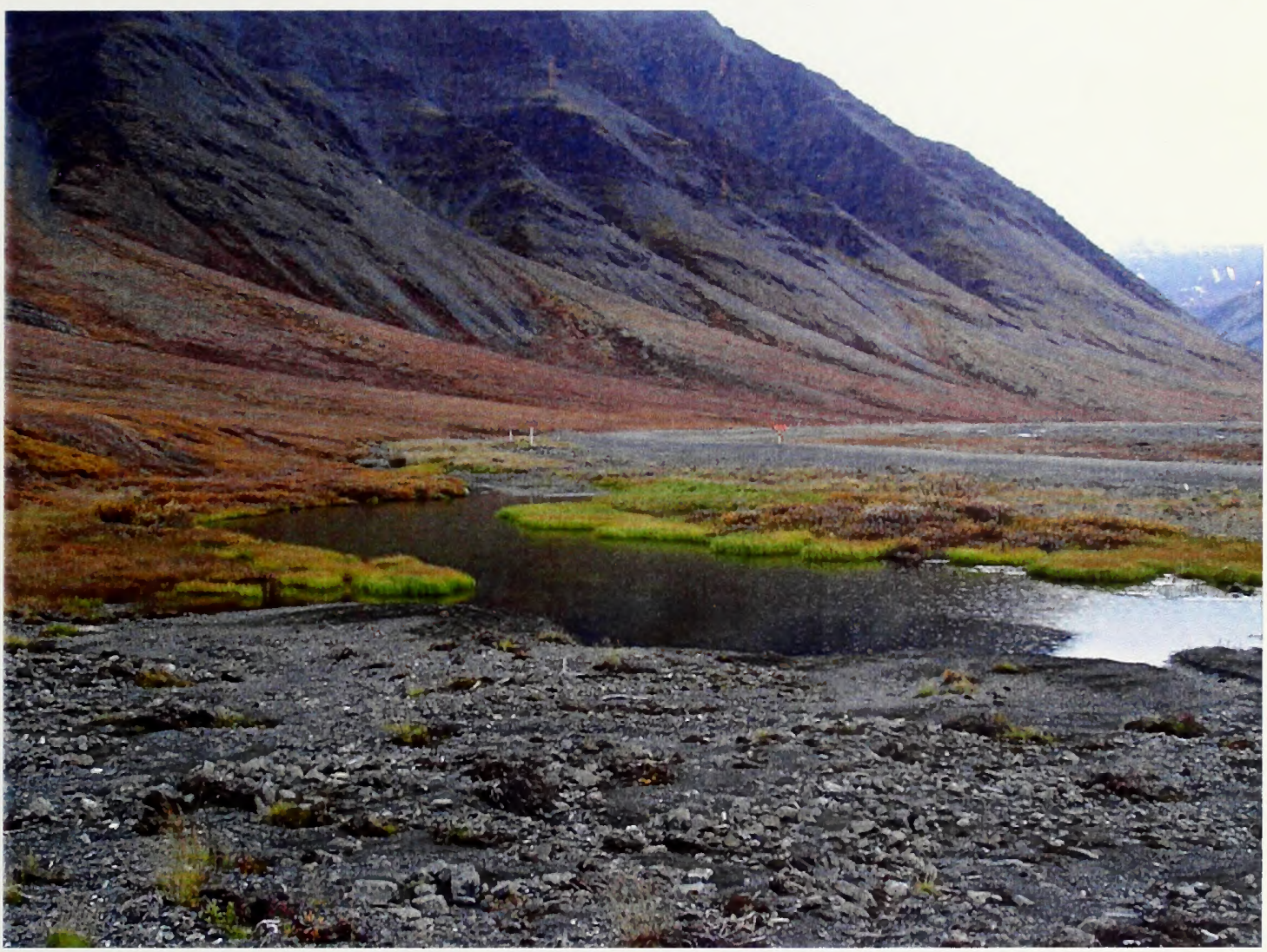




\section{The BLM Mission}

The Bureau of Land Management sustains the health, diversity and productivity of the public lands for the use and enjoyment of present and future generations.

\section{Cover Photo}

This pond, adjacent to the upper Atigun River drainage north of the Brooks Range, is ice-free all winter because of the piepline's thermal regime. It is used by Arctic grayling for rearing and overwintering. Alyeska Pipeline Service Company constructed a channel between the pond and the Atigun River so fish can freely use the pond all year. Photo by Dennis Gnath.

\section{Open File Reports}

Open File Reports issued by the Bureau of Land Management-Alaska present the results of inventories or other investigations on a variety of scientific and technical subjects that are made available to the public outside the formal BLM-Alaska technical publication series. These reports can include preliminary or incomplete data and are not published and distributed in quantity.

This report is available from the Office of Pipeline Monitoring, 411 West 4th Avenue, Anchorage, Alaska 99501, telephone (907) 257-1300.

Other reports in the series are available while supplies last from BLM External Affairs, 222 West 7th Avenue \#13, Anchorage, Alaska 99513 and from the Juneau Minerals Information Center, 100 Savikko Road, Mayflower Island, Douglas, AK 99824, (907) 364-1553. Copies are also available for inspection at the Alaska Resource Library and Information Service (Anchorage), the USDI Resources Library in Washington, D. C., various libraries of the University of Alaska, the BLM National Business Center Library (Denver) and other selected locations.

A complete bibliography of all BLM-Alaska scientific reports can be found on the Internet at: http://www.ak.blm.gov/aliairs/sci_rpts.himl. Related publications are also listed athitp://juneau.ak.blm.gov. 


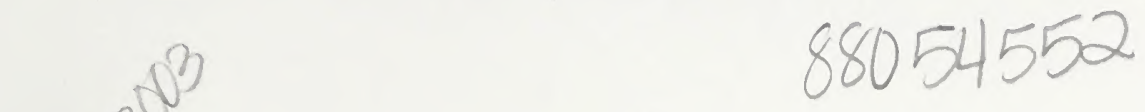

ф

\section{Zones of Restricted Activity for Protection of Key Fish Areas Along the Trans-Alaska Pipeline System on Federally-Administered Lands}

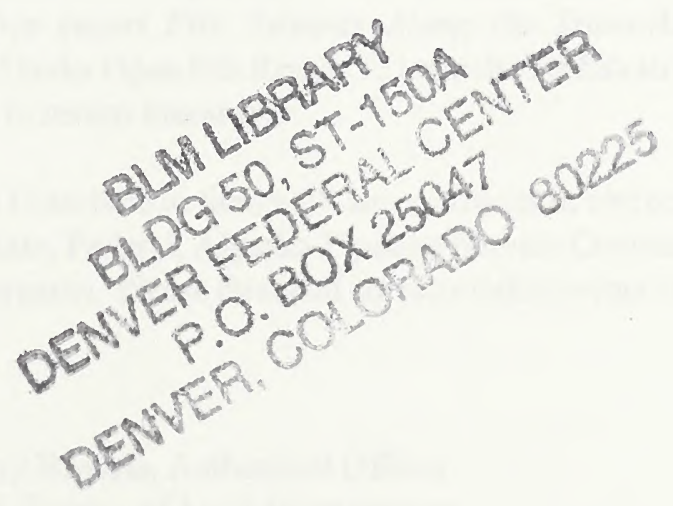

BLM-Alaska Open File Report 92 Seplember 2003

U. S. Department of the Interior Bureau of Land Management 
(1)

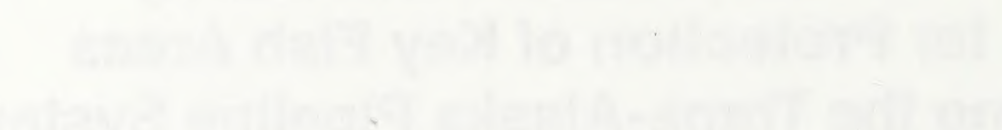

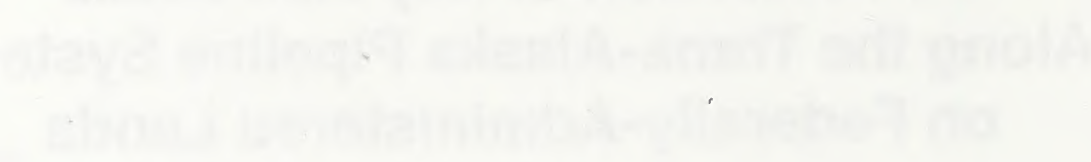


Preface for the Second Edition

The Agreement and Grant of Right-of-Way for Trans-Alaska Pipeline between the United States of America and Amerada Hess Corporation, ARCO Pipe Line Company, BP Pipelines Inc., Exxon Pipeline Company, Mobile Alaska Company, Phillips Petroleum Company, SOHIO Pipe Line Company, and Union Alaska PipeLine Company, Stipulation 2.5.3. Zones of Restricted Activities statcs:

“2.5.3.1. Permittees' activities in connection with the Pipeline System in key fish and wildlife areas may be restricted by the Authorized Officer during periods of fish and wildlife breeding, nesting, spawning, lambing or calving activity and during major migrations of fish and wildlife. The Authorized Officer shall give Permittees written notice of such restrictive action. From time to time, the Authorized Officer shall furnish Permittees a list of areas where such actions may be required, together with the anticipated dates of restriction.,

This document is the Federal Authorized Officer's list described, as above. As the Federal Authorized Officer's list, it includes only sensitive fishery habitats on Federally administered portions of the pipeline.

Sensitive fish streams listed in this report have been identified from another Office of Pipeline Office report Fish Streams Along the Trans-Alaska Pipeline System (BLM-Alaska Open File Report 93) which lists fish streams along TAPS mentioned in recent literature.

This list will be updated from time to time with any additions or corrections resulting from further State, Federal, Alyeska Pipeline Service Company or other study of pipeline streams. Please direct all correspondence concerning this report to:

Jerry Brossia, Authorized Officer

U.S. Bureau of Land Management

Office of Pipeline Monitoring

411 West $4^{\text {th }}$ Avenue

Anchorage, Alaska 99501

(907) $257-1300$ 



\section{Fish Species Codes}

(Adapted from Johnson and Rockwell, 1981)

:Dallia pectoralis

$\mathrm{AB}=$ Alaska blackfish

$\mathrm{AC}=$ Arctic char

$\mathrm{AL}=$ Arctic lamprey

AS $=$ American shad

$\mathrm{BB}=$ Burbot

$\mathrm{BC}=$ Bering cisco

$\mathrm{BL}=$ American brook lamprey

$\mathrm{BW}=$ Broad whitefish

$\mathrm{CA}=$ Arctic cisco

$\mathrm{CD}=$ Sculpin

$\mathrm{CI}=$ Cisco

$\mathrm{CN}=\quad$ Slimy sculpin

$\mathrm{CS}=$ Least cisco

$\mathrm{CT}=$ Cutthroat trout

DS $=$ Dog (Chum) salmon

DV $=$ Dolly varden

$\mathrm{GR}=$ Grayling

$\mathrm{HO}=$ Pond Smelt

$\mathrm{HW}=$ Humpback whitefish

IN $=$ Inconnu (Sheefish)

$\mathrm{KO}=$ Kokanee

$\mathrm{KS}=$ King (Chinook) salmon

LC = Lakc chub

LS $=$ Longnose sucker

LT = Lake trout

$\mathrm{LW}=$ Lake whitefish

$\mathrm{NP}=$ Northern pike

$\mathrm{OM}=$ Rainbow smelt

PS $=$ Pink (Humpback) salmon

$\mathrm{PW}=$ Pygmy whitefish

$\mathrm{RB}=$ Rainbow trout

RS = Red (Sockeye) salmon

$\mathrm{RW}=$ Round whitefish

SB $=$ Stickleback

S9 $=$ Ninespine stickleback

$\mathrm{SH}=$ Steelhead trout

SK $=$ Sucker

SS $=$ Coho (Silver) salmon

$\mathrm{TP}=$ Trout - Perch

WF $=$ Whitefish

:Salvelinus alpinus

:Lampetra iaponica

: Alosa sapidissima

:Lota lota

:Coregonus laurettac

:Lampetra sp.

:Coregonus nasus

:Coregonus autumnalis

Family Cottidae

:Coregonus sp.

:Cottus cognatus

:Coregonus sardinella

:Oncorhynchous clarkii

:Oncorhynchus keta

: Salvelinus malma

:Thymallus arcticus

:Hypomesus olidus

:Coregonus pidschian

:Stenodus leucichthys

:Oncorhynchus nerka

:Ohncorhynchus tshawytscha

:Couesius plumbeus

:Catostomus catostomus

:Salvelinus namaycush

:Coregomis clupeaformis

:Esox lucius

:Osmerus mordax

:Oncorhychus gorbuscha

:Prosopium coulteri

:Oncorhynchus mykiss

:Oncorhynchus nerka

:Prosopium cylindraceum

:Family Gasterosteidae

:Pungitius pungitius

:Oncorhynchus mykiss

:Family Catostomidae

:Oncorhynchus kisutch

:Percopsis omiscomaycus

:Coregonus sp. 



\section{EXPLANATION OF HEADINGS}

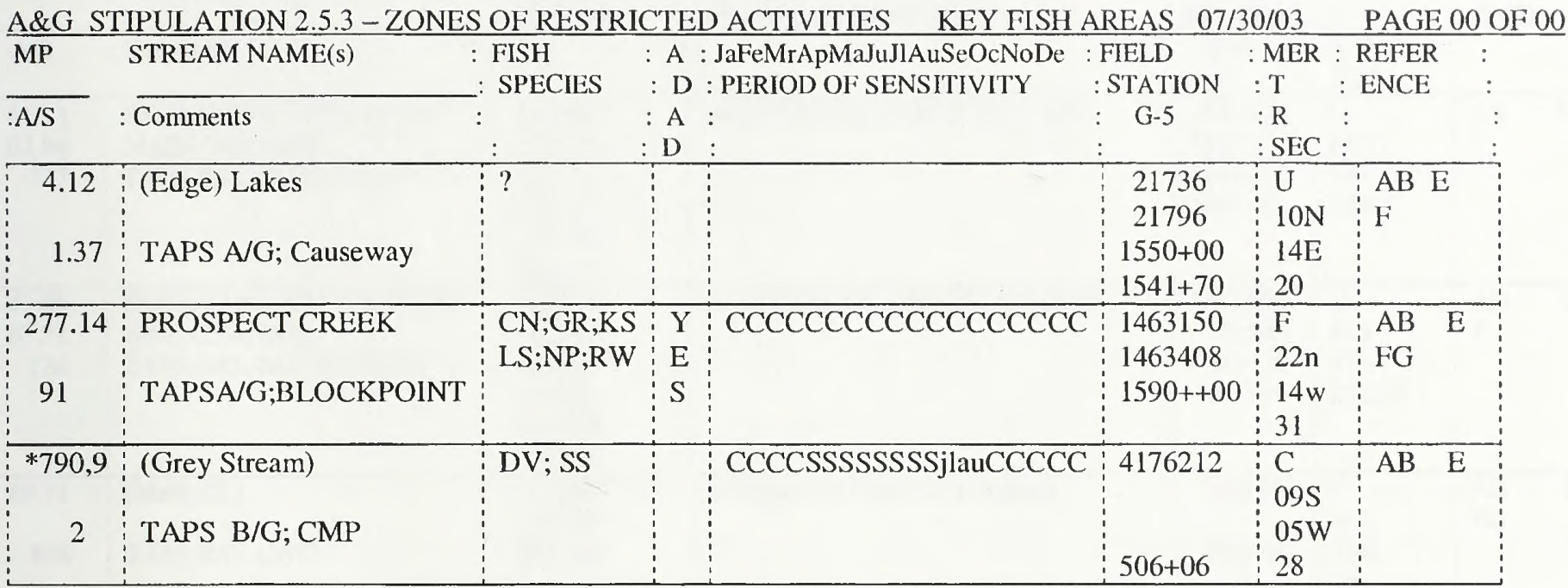

MP = The distance in miles from Pump Station 1; * Prefix denotes extrapolated mileage not field checked.

AS = Alyeska Pipeline Service Company (G-100 as-builts) alignment sheet number.

STREAM NAME = Adapted from Johnson and Rockwell, 1981. For example: YUKON RIVER denotes a name recognized by the U.S. Geological Survey; (Small or Jackie's CK) denotes a non-USGS recognized popular name; [Snowpad CK denotes a new name used in this list.

COMMENTS = TAPS A/G denotes above-ground pipe mode; TAPS B/G denotes below-ground mode; CMP is a corrugated-metal-pipe or culvert; LWC is a low water crossing; BLOCKPOINT is a physical barrier to vehicle passage; CAUSEWAY, BRIDGE, and PARALLEL are self-explanatory.

FISH SPECIES = see Page ii of iv for explanation of codes.

$\mathrm{ADAD}=$ "YES" denotes anadromous fish stream designated by Alaska Dept. Fish and Game.

PERIOD OF SENSITIVITY = C denotes Critical period of fish usage; $\mathrm{S}$ denotes Sensitive period of fish usage.

NOTE: UNDERLINING of a stream's period of sensitivity denotes the recommended sensitivity period if fish return in the future.

FIELD STATION = Distance in feet from Pump Station No 1 as estimated in field; * Prefix denotes an extrapolated stationing not field checked.

G-5 = Obtained from selected references (see Page iii of iv) and refers to construction drawings.

$\mathrm{MER}=$ Meridian $-\mathrm{U}$ is Umiat; $\mathrm{F}$ is Fairbanks; $\mathrm{C}$ is Copper River.

$\mathrm{T}=$ Tier or Township; $\mathrm{R}=$ Range; Sec. $=$ Section .

REFERENCE $=$ See page iii of iv. 
A\&G STIPULATION 2.5.3 ZONES OF RESTRICTED ACTIVITIES - KEY FISH AREAS $\quad 07 / 30 / 03 \quad$ PAGE 1 of 21

\begin{tabular}{|c|c|c|c|c|c|c|}
\hline MP & $\begin{array}{l}\text { : STREAM NAME(s) } \\
: \text { Comments }\end{array}$ & $\begin{array}{l}\text { FISH } \\
\text { SPECIES }\end{array}$ & $\begin{array}{l}\text { A: JaFeMrApMaJuJlAuSeOcNoDe } \\
\text { D : PERIOD OF SENSITIVITY } \\
\text { A : } \\
\text { D : }\end{array}$ & $\begin{array}{l}\text { FIELD } \\
\text { STATION } \\
\text { G-5 }\end{array}$ & $\begin{array}{l}: \text { MER } \\
: T \\
: \text { R } \\
: \text { SEC }\end{array}$ & $\begin{array}{l}\text { REFER } \\
- \text { ENCE }\end{array}$ \\
\hline $\begin{array}{r}61.93 \\
62.94 \\
127\end{array}$ & $\begin{array}{l}\text { SAGAVANIRKTOK RIVER } \\
\text { MAIN CHANNEL } \\
\text { TAPS B/G; BLOCKPOINT }\end{array}$ & $\begin{array}{l}\mathrm{AC}: \mathrm{BB} ; \\
\mathrm{BW} ; \mathrm{CA} ; \\
\mathrm{CN} ; \mathrm{CS} \\
\text { DS;GR; } \\
\text { HW?PS; } \\
\text { RW;S9 }\end{array}$ & SSSSSSSSCCCCSSCCCCCCSSSS & $\begin{array}{r}327000 \\
* 332329 \\
1197+12 \\
1143+83\end{array}$ & $\begin{array}{l}\mathrm{U} \\
01 \mathrm{~N} \\
14 \mathrm{E} \\
26 \& 27\end{array}$ & $\begin{array}{l}\mathrm{AB} \quad \mathrm{E} \\
\mathrm{F}\end{array}$ \\
\hline $\begin{array}{r}67.08 \\
67.71 \\
126\end{array}$ & $\begin{array}{l}\text { SAGAVANIRKTOK RIVER } \\
\text { SIDE CHANNEL } \\
\text { TAPS B/G; BLOCKPOINT }\end{array}$ & $\begin{array}{l}\text { AC:BB; } \\
\text { BW;CA; } \\
\text { CN;CS } \\
\text { DS;GR; } \\
\text { HW?PS; } \\
\text { RW;S9 }\end{array}$ & SSSSSSSSCCCCSSCCCCCCSSSS & $\begin{array}{r}* 354159 \\
357500 \\
933+28 \\
897+87\end{array}$ & $\begin{array}{l}U \\
01 S \\
14 \mathrm{E} \\
22 \& 23\end{array}$ & $\begin{array}{l}\mathrm{AB} \\
\mathrm{F}\end{array}$ \\
\hline $\begin{array}{r}69.71 \\
126\end{array}$ & $\begin{array}{l}\text { (Mark CK) } \\
\text { TAPS B/G; LWC }\end{array}$ & $\begin{array}{l}\text { AC;BB } \\
\text { CN;GR; } \\
\text { RW;S9? }\end{array}$ & jafemrapCCCCSSCCCCocnode & $\begin{array}{l}368058 \\
792+00\end{array}$ & $\begin{array}{l}\mathrm{U} \\
01 \mathrm{~S} \\
14 \mathrm{E} \\
34\end{array}$ & $\begin{array}{l}\mathrm{AB} \\
\mathrm{FG}\end{array}$ \\
\hline $\begin{array}{r}69.77 \\
126\end{array}$ & $\begin{array}{l}\text { SAGAVANIRKTOK RIVER } \\
\text { SIDE CHANNEL } \\
\text { TAPS B/G; BLOCKPOINT }\end{array}$ & $\begin{array}{l}\text { AC;CN? } \\
\text { GR;S9 }\end{array}$ & jafemrapSSSSSSSSSSSSnode & $\begin{array}{l}368400 \\
791+00 \\
790+40 \\
\end{array}$ & $\begin{array}{l}U \\
01 S \\
14 \mathrm{E} \\
34\end{array}$ & $\begin{array}{l}\text { ABEFG } \\
\text { F }\end{array}$ \\
\hline $\begin{array}{r}70.54 \\
126\end{array}$ & $\begin{array}{l}\text { SAGAVANIRKTOK RIVER } \\
\text { SIDE CHANNEL } \\
\text { TAPS B/G; LWC }\end{array}$ & $\begin{array}{l}\text { AC;CN"? } \\
\text { GR;S9 }\end{array}$ & jafemrapSSSSSSSSSSSSnode & $\begin{array}{l}372450 \\
747+12\end{array}$ & $\begin{array}{l}U \\
02 S \\
14 E \\
03\end{array}$ & $\begin{array}{l}\mathrm{AB} \\
\mathrm{F}\end{array}$ \\
\hline $\begin{array}{r}70.81 \\
125\end{array}$ & $\begin{array}{l}\text { SAGAVANIRKTOK RIVER } \\
\text { SIDE CHANNEL } \\
\text { TAPS B/G; LWC }\end{array}$ & $\begin{array}{l}\mathrm{AC} ; \mathrm{CN} ? \\
\mathrm{GR} ; \mathrm{S} 9\end{array}$ & jafemrapSSSSSSSSSSSSnode & $\begin{array}{l}373875 \\
734+30\end{array}$ & $\begin{array}{l}U \\
02 S \\
14 \mathrm{E} \\
04\end{array}$ & $\begin{array}{l}\mathrm{AB} \\
\mathrm{F}\end{array}$ \\
\hline $\begin{array}{r}72.06 \\
125\end{array}$ & $\begin{array}{l}\text { SAGAVANIRKTOK RIVER } \\
\text { SIDE CHANNEL } \\
\text { TAPS B/G; LWC }\end{array}$ & $\begin{array}{l}\text { AC?CN? } \\
\text { GR?S9? }\end{array}$ & jafemrapSSSSSSSSSSSSnode & $\begin{array}{l}380500 \\
666+00\end{array}$ & $\begin{array}{l}U \\
02 S \\
14 \mathrm{E} \\
16\end{array}$ & $\begin{array}{l}\mathrm{AB} \\
\mathrm{F}\end{array}$ \\
\hline $\begin{array}{r}75.34 \\
125\end{array}$ & $\begin{array}{l}\text { SAGAVANIRKTOK RIVER } \\
\text { SIDE CHANNEL } \\
\text { (Spoiled Mary CK ) } \\
\text { TAPS B/G; LWC }\end{array}$ & $\begin{array}{l}\text { AC;CN; } \\
\text { GR }\end{array}$ & jafemrapCCCCSSCCCCocnode & $\begin{array}{l}397819 \\
492+00\end{array}$ & $\begin{array}{l}\mathrm{U} \\
02 \mathrm{~S} \\
14 \mathrm{E} \\
33\end{array}$ & $\begin{array}{l}\mathrm{AB} \\
\mathrm{G}\end{array}$ \\
\hline $\begin{array}{r}76.04 \\
76.41 \\
125 \\
124\end{array}$ & $\begin{array}{l}\text { SAGAVANIRKTOK RIVER } \\
\text { SIDE CHANNEL } \\
\text { TAPS B/G; BLOCKPOINT }\end{array}$ & $\begin{array}{l}\text { AC;BB; } \\
\text { BW;CA; } \\
\text { CN;CS;DS } \\
\text { GR;HW? } \\
\text { PS;RW;S9 }\end{array}$ & SSSSSSSSCCCCSSCCCCCCSSSS & $\begin{array}{l}401498 \\
403455 \\
463+00 \\
446+00\end{array}$ & $\begin{array}{l}U \\
02 S \\
14 \mathrm{E} \\
33\end{array}$ & $\begin{array}{ll:l}\mathrm{U} & : & : \mathrm{A} \\
03 \mathrm{~S}: & : \mathrm{B} & \\
14 \mathrm{E}: & : \mathrm{E} & \\
33: & : \mathrm{F} & \end{array}$ \\
\hline $\begin{array}{r}79.94 \\
124\end{array}$ & $\begin{array}{l}\text { (Woody CK) } \\
\text { TAPS B/G; LWC }\end{array}$ & GR & jafemrapSSSSSSSSSSocnode & $\begin{array}{l}* 422115 \\
256+83\end{array}$ & \begin{tabular}{|l}
$U$ \\
$03 \mathrm{~S}$ \\
$14 \mathrm{E}$ \\
20 \\
\end{tabular} & $\begin{array}{l}\mathrm{AB} \\
\mathrm{F}\end{array}$ \\
\hline $\begin{array}{r}80.34 \\
124\end{array}$ & $\begin{array}{l}\text { SAGAVANIRKTOK RIVER } \\
\text { SIDE CHANNEL } \\
\text { TAPS B/G; LWC }\end{array}$ & AC? GR & jafemrapCCCCSSauseocnode & $\begin{array}{l}* 424180 \\
236+18\end{array}$ & $\begin{array}{l}\mathrm{U} \\
03 \mathrm{~S} \\
14 \mathrm{E} \\
20 \\
\end{array}$ & $\mathrm{~F}^{\mathrm{B}}$ \\
\hline $\begin{array}{r}83.25 \\
84.22 \\
123\end{array}$ & $\begin{array}{l}\text { SAGAVANIRKTOK RIVER } \\
\text { SIDE CHANNEL } \\
\text { TAPS B/G; BLOCKPOINT }\end{array}$ & $\begin{array}{l}\mathrm{AC} ; \mathrm{BB} ; \\
\text { BW;CA; } \\
\text { CN;CS } \\
\text { DS;GR; } \\
\text { HW?PS: } \\
\text { RW;S9 }\end{array}$ & $\begin{array}{l:l} & \text { SSSSSSSSCCCCSSCCCCCCSSSS } \\
E_{1} & \\
S & \end{array}$ & $\begin{array}{r}* 439572 \\
444700 \\
82+26 \\
58+15\end{array}$ & $\begin{array}{l}\mathrm{U} \\
04 \mathrm{~S} \\
14 \mathrm{E} \\
05,07 \& 08\end{array}$ & $\begin{array}{ll}\mathrm{AB} & \mathrm{E} \\
\mathrm{F} & \end{array}$ \\
\hline
\end{tabular}


A\&G STIPULATION 2.5.3 ZONES OF RESTRICTED ACTIVITIES - KEY FISH AREAS 07/30/03 PAGE 2 of 21

\begin{tabular}{|c|c|c|c|c|c|c|}
\hline MP & : STREAM NAME(s) & $\begin{array}{l}\text { FISH } \\
\text { SPECIES }\end{array}$ & $\begin{array}{l}\text { A: JaFeMrApMaJuJlAuSeOcNoDe } \\
\text { D : PERIOD OF SENSITIVITY } \\
\text { A : } \\
\text { D : }\end{array}$ & $\begin{array}{l}\text { FIELD } \\
\text { STATION } \\
\quad \text { G-5 }\end{array}$ & $\begin{array}{l}: \text { MER } \\
: \mathrm{T} \\
: \mathrm{R} \\
: \text { SEC }\end{array}$ & $\begin{array}{l}: \text { REFER } \\
: \text {-ENCE } \\
:\end{array}$ \\
\hline 84.96 & $\begin{array}{l}\text { (Dan or Charlotte CK) } \\
\text { TAPS A/G; BRIDGE \& } 2 \text { CMPs }\end{array}$ & $\begin{array}{l}\mathrm{AC} ; \mathrm{CN} ; \\
\mathrm{GR} ; \mathrm{RW} \\
\mathrm{S} 9\end{array}$ & $\begin{array}{ccc}Y & \text { jafemrapCCCCSSCCCCocnode } \\
\mathrm{E} & 448439 \mathrm{BRE} 448509 \\
\mathrm{~S} & 448509 \mathrm{CMP} 448559 \\
& 448679 \text { CMP448739 }\end{array}$ & $\begin{array}{r}448439 \\
448739 \\
20+12\end{array}$ & $\begin{array}{l}\mathrm{U} \\
04 \mathrm{~S} \\
14 \mathrm{E} \\
08\end{array}$ & $\begin{array}{l}\mathrm{AB} \\
\mathrm{FG}\end{array}$ \\
\hline $\begin{array}{r}86.94 \\
123\end{array}$ & $\begin{array}{l}\text { (Lori CK) } \\
\text { TAPS A/G; LWC }\end{array}$ & $\overline{G R}$ & $\begin{array}{ll}\text { jafemrapSSSSSSSSSSSocnode } \\
\end{array}$ & $\begin{array}{r}459029 \\
459099 \\
1722+00\end{array}$ & $\begin{array}{l}\mathrm{U} \\
04 \mathrm{~S} \\
14 \mathrm{E} \\
29\end{array}$ & $\begin{array}{l}A B \\
F G\end{array}$ \\
\hline $\begin{array}{r}91.93 \\
122\end{array}$ & $\begin{array}{l}\text { (Stump CK) } \\
\text { TAPS A/G; LWC }\end{array}$ & $\begin{array}{l}\text { CN;GR; } \\
\text { LT;S9 }\end{array}$ & jafemrapSSSSSSSSSSocnode & $\begin{array}{r}485366 \\
485425 \\
1499+00\end{array}$ & $\begin{array}{l}U \\
05 S \\
14 \mathrm{E} \\
16 \\
\end{array}$ & $\begin{array}{r}\mathrm{B} \\
\mathrm{FG}\end{array}$ \\
\hline $\begin{array}{c}92.19 \\
92.36 \\
122\end{array}$ & $\begin{array}{l}\text { (Clarke's Lake) } \\
\text { TAPS A/G; Causeway \& CMP }\end{array}$ & $\begin{array}{l}\text { CN;GR; } \\
\text { LT;S9 }\end{array}$ & CCCCCCCCCCCCSSSSCCCCCCCC & $\begin{array}{r}486739 \\
487636 \\
1489+28 \\
1481+00 \\
\end{array}$ & $\begin{array}{l}U \\
05 S \\
14 \mathrm{E} \\
16 \\
\end{array}$ & $\begin{array}{l}\mathrm{AB} \\
\mathrm{FG}\end{array}$ \\
\hline $\begin{array}{r}92.97 \\
122\end{array}$ & $\begin{array}{l}\text { SAGAVANIRKTOK RIVER } \\
\text { SIDE CHANNEL } \\
\text { TAPS A/G; LWC }\end{array}$ & $\begin{array}{l}\text { AC? CN; } \\
\text { GR;RW; } \\
\text { S9? }\end{array}$ & jafemrapCCCCSSSCCSSSnode & $\begin{array}{r}490853 \\
490922 \\
1445+25\end{array}$ & $\begin{array}{l}\mathrm{U} \\
05 \mathrm{~S} \\
14 \mathrm{E} \\
21\end{array}$ & $\begin{array}{l}\text { A B } \\
\text { F }\end{array}$ \\
\hline $\begin{array}{r}93.36 \\
122\end{array}$ & $\begin{array}{l}\text { SAGAVANIRKTOK RIVER } \\
\text { SIDE CHANNEL } \\
\text { TAPS B/G; LWC }\end{array}$ & $\begin{array}{l}\text { AC? CN? } \\
\text { GR;S9 }\end{array}$ & jafemrapCCCCSSSCCSSSnode & $\begin{array}{r}492917 \\
492973 \\
1424+79\end{array}$ & \begin{tabular}{|l}
$\mathrm{U}$ \\
$05 \mathrm{~S}$ \\
$14 \mathrm{E}$ \\
21 \\
\end{tabular} & $\begin{array}{l}\mathrm{AB} \\
\mathrm{F}\end{array}$ \\
\hline $\begin{array}{r}95.79 \\
121\end{array}$ & $\begin{array}{l}\text { (Arthur CK) } \\
\text { TAPS A/G; LWC }\end{array}$ & $\begin{array}{l}\mathrm{AC} ; \mathrm{BB} \\
\mathrm{CN} ; \mathrm{GR}\end{array}$ & jafemrapCCCCSSSCCCocnode & $\begin{array}{r}505760 \\
505825 \\
1297+50\end{array}$ & $\begin{array}{l}U \\
05 S \\
14 \mathrm{E} \\
32 \\
\end{array}$ & $\begin{array}{l}\mathrm{AB} \\
\mathrm{FG}\end{array}$ \\
\hline $\begin{array}{r}96.12 \\
121\end{array}$ & $\begin{array}{l}\text { (Gustafson Gulch) } \\
\text { TAPS A/G; LWC }\end{array}$ & $\begin{array}{l}\mathrm{AC} ; \mathrm{BB} ; \\
\mathrm{CN} ; \mathrm{GR}\end{array}$ & jafemrapCCCCSSSCCCocnode & $\begin{array}{r}507485 \\
507550 \\
1280+00\end{array}$ & $\begin{array}{l}\mathrm{U} \\
06 \mathrm{~S} \\
14 \mathrm{E} \\
05\end{array}$ & $\begin{array}{l}\mathrm{AB} \\
\mathrm{FG}\end{array}$ \\
\hline $\begin{array}{r}99.07 \\
121\end{array}$ & $\begin{array}{l}\text { (Polygon CK) } \\
\text { TAPS A/G; LWC }\end{array}$ & $\begin{array}{l}\mathrm{AC} ; \mathrm{BB} ; \\
\mathrm{CN} ; \mathrm{GR}\end{array}$ & jafemrapCCCCSSSCCCocnode & $\begin{array}{r}523070 \\
523145 \\
1125+03\end{array}$ & $\begin{array}{l}U \\
06 S \\
14 \mathrm{E} \\
19 \\
\end{array}$ & $\begin{array}{l}\mathrm{AB} \\
\text { FG }\end{array}$ \\
\hline 99.99 & $\begin{array}{l}\text { (Poison Pipe CK) } \\
\text { TAPS A/G; LWC }\end{array}$ & $\begin{array}{l}\mathrm{AC} ; \mathrm{CN} \\
\mathrm{GR}\end{array}$ & jafemrapCCCCSSSCCSSSocnode & $\begin{array}{r}527926 \\
527986 \\
1077+10\end{array}$ & $\begin{array}{l}U \\
06 S \\
14 \mathrm{E} \\
19 \\
\end{array}$ & $\begin{array}{l}\mathrm{AB} \\
\mathrm{FG}\end{array}$ \\
\hline $\begin{array}{c}100.31 \\
120\end{array}$ & $\begin{array}{l}\text { (Climb CK) } \\
\text { TAPS A/G; CMP }\end{array}$ & $\mathrm{AC} ; \mathrm{GR}$ & jafemrapCCCCSSSCCCocnode & $\begin{array}{r}529599 \\
529669 \\
1060+34\end{array}$ & $\begin{array}{ll}U \\
06 \mathrm{~S} \\
14 \mathrm{E} \\
30 \\
\end{array}$ & $\begin{array}{l}\mathrm{AB} \\
\mathrm{FG}\end{array}$ \\
\hline $\begin{array}{c}100.82 \\
120\end{array}$ & $\begin{array}{l}\text { (Dennis CK) } \\
\text { TAPS A/G; LWC }\end{array}$ & $\mathrm{AC} ; \mathrm{GR}$ & jafemrapSSSSSSSSSSocnode & $\begin{array}{r}532280 \\
532345 \\
1033+60\end{array}$ & \begin{tabular}{|l} 
\\
$06 \mathrm{~S}$ \\
$14 \mathrm{E}$ \\
30 \\
\end{tabular} & $\begin{array}{l}\mathrm{AB} \\
\mathrm{FG}\end{array}$ \\
\hline $\begin{array}{c}102.45 \\
120\end{array}$ & $\begin{array}{l}\text { (Rudy CK) } \\
\text { TAPS A/G; LWC }\end{array}$ & $\begin{array}{l}\mathrm{AC} ; \mathrm{CN} \\
\mathrm{GR}\end{array}$ & jafemrapCCCCSSSCCCocnode & $\begin{array}{l}540899 \\
540969 \\
949+99\end{array}$ & \begin{tabular}{|l}
$U$ \\
$07 \mathrm{~S}$ \\
$14 \mathrm{E}$ \\
05 \\
\end{tabular} & $\begin{array}{l}\mathrm{AB} \\
\mathrm{FG}\end{array}$ \\
\hline
\end{tabular}


A\&G STIPULATION 2.5.3 ZONES OF RESTRICTED ACTIVITIES - KEY FISH AREAS 07/30/03 PAGE 3 of 21

\begin{tabular}{|c|c|c|c|c|c|c|}
\hline MP & STREAM NAME(s) & $\begin{array}{l}\text { FISH } \\
\text { SPECIES }\end{array}$ & $\begin{array}{l}\text { A: JaFeMrApMaJuJlAuSeOcNoDe } \\
\text { D : PERIOD OF SENSITIVITY }\end{array}$ & $\begin{array}{l}\text { FIELD } \\
\text { STATION }\end{array}$ & $\begin{array}{l}\text { MER } \\
T\end{array}$ & $\begin{array}{l}\text { REFER } \\
\text {-ENCE }\end{array}$ \\
\hline $\mathrm{A} / \mathrm{S}$ & Comments & & $\begin{array}{l}\text { A : } \\
\text { D : }\end{array}$ & G-5 & & \\
\hline 120 & TAPS A/G; BLOCKPOINT & GR;RW? & & $895+76$ & $\begin{array}{l}14 \mathrm{E} \\
08\end{array}$ & \\
\hline $\begin{array}{c}113.52 \\
119\end{array}$ & $\begin{array}{l}\text { (Thiele's Trickle) } \\
\text { TAPS A/G; LWC }\end{array}$ & GR; & jafemrapSSSSSSSSSSSSocnode & $\begin{array}{r}599383 \\
599438 \\
1513+06\end{array}$ & $\begin{array}{l}\mathrm{U} \\
08 \mathrm{~S} \\
14 \mathrm{E} \\
23\end{array}$ & $\begin{array}{ll}\mathrm{AB} & \mathrm{E} \\
\mathrm{F} & \end{array}$ \\
\hline $\begin{array}{c}117.11 \\
118\end{array}$ & $\begin{array}{l}\text { (Oksrukuyik CK) } \\
\text { TAPS A/G; BLOCKPOINT }\end{array}$ & $\begin{array}{l}\mathrm{AC} ; \mathrm{BB} ? \\
\mathrm{CN} ; \mathrm{GR} ; \\
\mathrm{LT} ; \mathrm{RW} ?\end{array}$ & jafemrapCCCCSSSCCCCCnode & $\begin{array}{r}618290 \\
618360 \\
1323+71\end{array}$ & $\begin{array}{l}\mathrm{U} \\
08 \mathrm{~S} \\
13 \mathrm{E} \\
32\end{array}$ & $\begin{array}{l}\mathrm{AB} \\
\mathrm{FG}\end{array}$ \\
\hline $\begin{array}{c}123.92 \\
117\end{array}$ & $\begin{array}{l}\text { TOOLIK RIVER } \\
\text { TAPS A/G; BLOCKPOINT }\end{array}$ & $\mathrm{AC} ; \mathrm{GR}$ & jafemrapSSSSSSSSSSSSnode & $\begin{array}{l}654304 \\
968+30\end{array}$ & $\begin{array}{l}\mathrm{U} \\
09 \mathrm{~S} \\
12 \mathrm{E} \\
16 \\
\end{array}$ & $\begin{array}{l}\mathrm{AB} \\
\mathrm{FG}\end{array}$ \\
\hline $\begin{array}{l}124.80 \\
117\end{array}$ & $\begin{array}{l}\text { (East Fork KUPARUK RIVER) } \\
\text { TAPS A/G; LWC }\end{array}$ & CN?GR & jafemrapSSSSSSSSSSSSnode & $\begin{array}{l}658915 \\
658985 \\
921+90\end{array}$ & $\begin{array}{l}\mathrm{U} \\
09 \mathrm{~S} \\
12 \mathrm{E} \\
17 \\
\end{array}$ & $\begin{array}{l}\mathrm{AB} \\
\mathrm{FG}\end{array}$ \\
\hline $\begin{array}{c}126.33 \\
117\end{array}$ & $\begin{array}{l}\text { KUPARUK RIVER } \\
\text { TAPS A/G; BLOCKPOINT }\end{array}$ & $\begin{array}{l}\text { AC?CN; } \\
\text { GR;LT? }\end{array}$ & jafemrapCCCCSSSCCCCCnode & $\begin{array}{l}666969 \\
667043 \\
842+00\end{array}$ & $\begin{array}{l}\mathrm{U} \\
09 \mathrm{~S} \\
12 \mathrm{E} \\
19\end{array}$ & $\begin{array}{l}\mathrm{AB} \\
\mathrm{FG}\end{array}$ \\
\hline $\begin{array}{c}127.04 \\
117\end{array}$ & $\begin{array}{l}\text { (Holt CK) } \\
\text { TAPS A/G; LWC }\end{array}$ & GR & jafemrapCCCCSSSCCCCCnode & $\begin{array}{l}670736 \\
670806 \\
804+36\end{array}$ & $\begin{array}{l}\mathrm{U} \\
09 \mathrm{~S} \\
11 \mathrm{E} \\
25\end{array}$ & $\begin{array}{l}\mathrm{AB} \\
\mathrm{FG}\end{array}$ \\
\hline $\begin{array}{l}127.17 \\
116\end{array}$ & $\begin{array}{l}\text { (Becky CK) } \\
\text { TAPS A/G; LWC }\end{array}$ & GR & jafemrapCCCCSSSCCCCCnode & $\begin{array}{l}671416 \\
671488 \\
799+82\end{array}$ & $\begin{array}{l}\mathrm{U} \\
09 \mathrm{~S} \\
11 \mathrm{E} \\
25 \\
\end{array}$ & $\begin{array}{l}\mathrm{AB} \\
\mathrm{FG}\end{array}$ \\
\hline $\begin{array}{c}128.62 \\
116\end{array}$ & $\begin{array}{l}\text { (Becky CK) } \\
\text { TAPS A/G; LWC }\end{array}$ & GR & jafemrapCCCCSSSCCCCCnode & $\begin{array}{l}679066 \\
679141 \\
721+20\end{array}$ & $\begin{array}{l}\mathrm{U} \\
09 \mathrm{~S} \\
11 \mathrm{E} \\
35\end{array}$ & $\begin{array}{l}\mathrm{AB} \\
\mathrm{FG}\end{array}$ \\
\hline $115 \mathrm{API}$ & $-3:($ Terry CK $)$ & & & & & B \\
\hline $\begin{array}{c}133.49 \\
115\end{array}$ & $\begin{array}{l}\text { (Terry CK) } \\
\text { TAPS A/G; LWC }\end{array}$ & AC?GR & jafemrapSSSSSSSSSSSSnode & $\begin{array}{l}704769 \\
704839 \\
465+91\end{array}$ & $\begin{array}{l}\mathrm{U} \\
10 \mathrm{~S} \\
11 \mathrm{E} \\
23\end{array}$ & $\begin{array}{l}\mathrm{AB} \\
\mathrm{FG}\end{array}$ \\
\hline $\begin{array}{c}134.01 \\
115\end{array}$ & $\begin{array}{l}\text { (Mack CK) } \\
\text { TAPS A/G; LWC }\end{array}$ & AC?;GR & jafemrapSSSSSSSSSSSSnode & $\begin{array}{l}707537 \\
707597 \\
438+29\end{array}$ & $\begin{array}{l}\mathrm{U} \\
10 \mathrm{~S} \\
11 \mathrm{E} \\
26 \\
\end{array}$ & $\begin{array}{l}\mathrm{AB} \\
\mathrm{FG}\end{array}$ \\
\hline $\begin{array}{c}134.33 \\
115\end{array}$ & $\begin{array}{l}(\mathrm{Ed} \mathrm{CK}) \\
\text { TAPS A/G; LWC }\end{array}$ & $\begin{array}{l}\mathrm{AC} ; \mathrm{GR} \\
\mathrm{LT} \text { ? }\end{array}$ & jafemrapSSSSSSSSSSSSnode & $\begin{array}{l}709205 \\
709275 \\
421+74\end{array}$ & $\begin{array}{l}\mathrm{U} \\
10 \mathrm{~S} \\
11 \mathrm{E} \\
26 \\
\end{array}$ & $\begin{array}{l}\mathrm{AB} \\
\mathrm{FG}\end{array}$ \\
\hline $\begin{array}{c}135.11 \\
115\end{array}$ & $\begin{array}{l}\text { (Jill CK) } \\
\text { TAPS A/G; LWC }\end{array}$ & AC?GR & jafemrapSSSSSSSSSSSSnode & $\begin{array}{l}713343 \\
713413 \\
380+60\end{array}$ & $\begin{array}{l}\mathrm{U} \\
10 \mathrm{~S} \\
11 \mathrm{E} \\
35 \\
\end{array}$ & $\begin{array}{l}\mathrm{AB} \\
\mathrm{FG}\end{array}$ \\
\hline $\begin{array}{c}142.02 \\
114\end{array}$ & $\begin{array}{l}\text { ATIGUN RIVER } \\
\text { TAPS A/G; BLOCKPOINT }\end{array}$ & $\begin{array}{l}\text { AC;BB; } \\
\text { CN;GR; } \\
\text { LT;RW }\end{array}$ & jafemrapCCCCSSSCCCCCSSSS & $\begin{array}{l}749548 \\
750162 \\
20+94\end{array}$ & $\begin{array}{l}\mathrm{U} \\
11 \\
12 \\
32\end{array}$ & $\begin{array}{l}\mathrm{AB} \\
\mathrm{FG}\end{array}$ \\
\hline $\begin{array}{r}143.23 \\
114 \\
\end{array}$ & $\begin{array}{l}\text { (Tee Lake Outlet) } \\
\text { TAPS A/G; LWC }\end{array}$ & $\begin{array}{l}\mathrm{AC} ; \mathrm{BB} ; \\
\mathrm{CN} ; \mathrm{GR} \\
\mathrm{GR} ; \mathrm{LT} ;\end{array}$ & jafemrapCCCCCCCCCCCCSSSS & $\begin{array}{l}756223 \\
756293 \\
155+29 \\
\end{array}$ & $\begin{array}{l}\mathrm{U} \\
12 \mathrm{~S} \\
12 \mathrm{E} \\
\end{array}$ & $\begin{array}{l}\mathrm{ABCDE} \\
\mathrm{FG}\end{array}$ \\
\hline
\end{tabular}




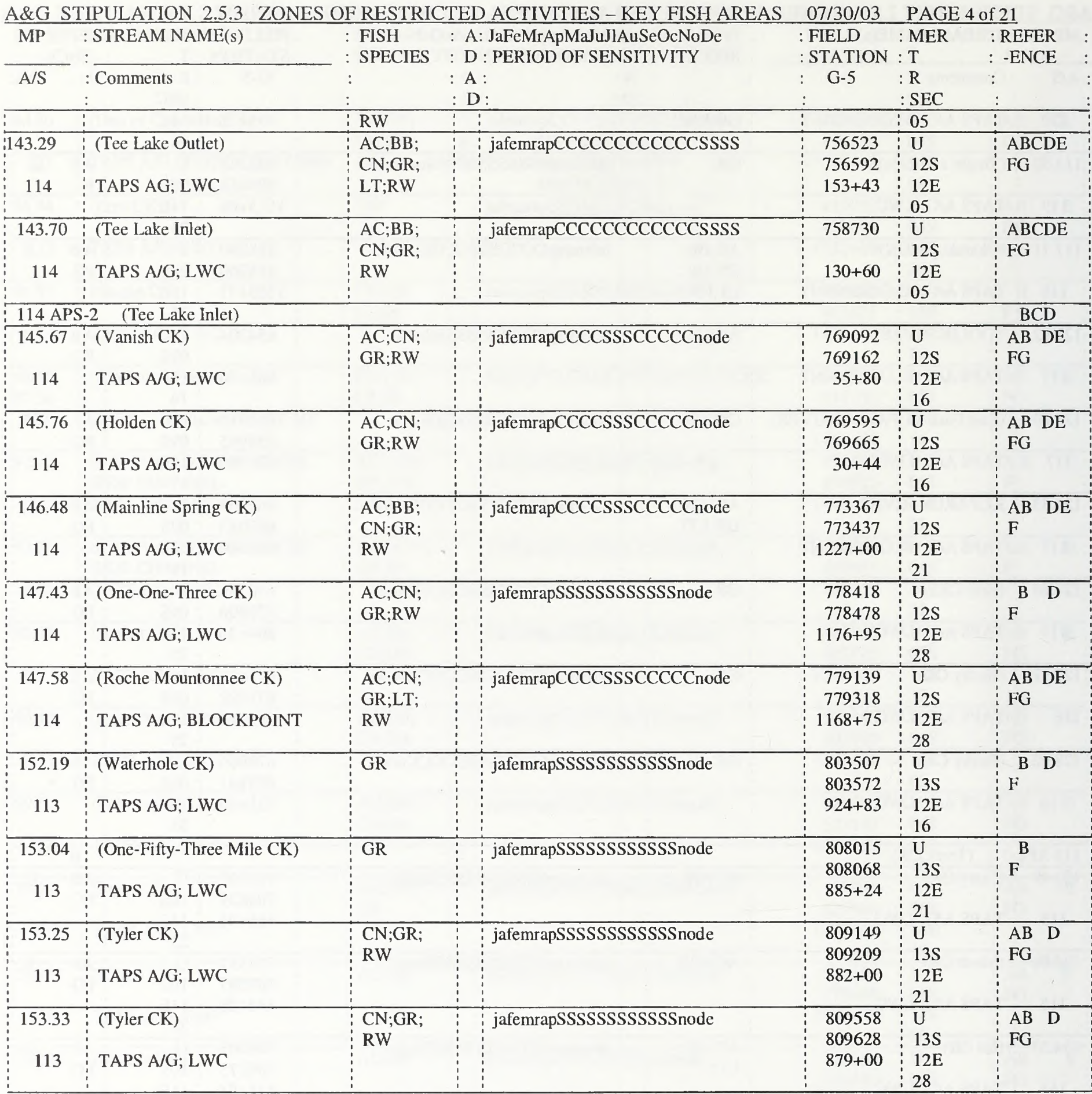

\begin{tabular}{|c|c|c|c|c|c|c|}
\hline $\begin{array}{c}153.47 \\
112\end{array}$ & $\begin{array}{l}\text { (One-One-Two CK) } \\
\text { TAPS A/G; LWC }\end{array}$ & GR & jafemrapSSSSSSSSSSSSnode & $\begin{array}{l}810288 \\
810353 \\
871+00\end{array}$ & $\begin{array}{l}\mathrm{U} \\
13 \mathrm{~S} \\
12 \mathrm{E} \\
28\end{array}$ & $\begin{array}{l}{ }_{\text {B }} \text { B D } \\
\text {. }\end{array}$ \\
\hline
\end{tabular}


A\&G STIPULATION 2.5.3 ZONES OF RESTRICTED ACTIVITIES - KEY FISH AREAS 07/30/03 PAGE 5 of 21

\begin{tabular}{|c|c|c|c|c|c|c|}
\hline MP & $\begin{array}{l}\text { STREAM NAME(s) } \\
\text { Comments }\end{array}$ & $\begin{array}{l}\text { F1SH } \\
: \text { SPECIES }\end{array}$ & $\begin{array}{l}\text { A: JaFeMrApMaJuJlAuSeOcNoDe } \\
\text { : D : PERIOD OF SENSITIVITY } \\
\text { : A : } \\
\text { : D : }\end{array}$ & $\begin{array}{l}: \text { FIELD } \\
: \text { STATION } \\
: \quad \text { G-5 } \\
\end{array}$ & $\begin{array}{l}\text { MER } \\
\text { T } \\
: \text { R } \\
: \text { SEC }\end{array}$ & $\begin{array}{l}\text { REFER } \\
\text {-ENCE }\end{array}$ \\
\hline $\begin{array}{c}153.59 \\
112\end{array}$ & $\begin{array}{l}\text { (Tyler CK) } \\
\text { TAPS A/G; LWC }\end{array}$ & $\begin{array}{l}\mathrm{CN} ; \mathrm{GR} \\
\mathrm{RW}\end{array}$ & jafemrapSSSSSSSSSSSSnode & $\begin{array}{l}810947 \\
811012 \\
860+00\end{array}$ & $\begin{array}{l}\mathrm{U} \\
13 \mathrm{~S} \\
12 \mathrm{E} \\
28\end{array}$ & $\begin{array}{l}\text { AB D } \\
\text { FG }\end{array}$ \\
\hline $\begin{array}{c}154.12 \\
112\end{array}$ & $\begin{array}{l}\text { (Trevor CK) } \\
\text { TAPS A/G; LWC }\end{array}$ & $\begin{array}{l}\mathrm{AC} ; \mathrm{CN} \\
\mathrm{GR} ; \mathrm{RW}\end{array}$ & jafemrapCCCCSSSCCCCCnode & $\begin{array}{l}813716 \\
813786 \\
837+00\end{array}$ & $\begin{array}{l}\mathrm{U} \\
13 \mathrm{~S} \\
12 \mathrm{E} \\
28\end{array}$ & $\begin{array}{ll}\mathrm{AB} & \mathrm{E} \\
\mathrm{F} & \end{array}$ \\
\hline $\begin{array}{l}157.25 \\
165.45 \\
112 \\
111 \\
110\end{array}$ & $\begin{array}{l}\text { ATIGUN RIVER \& } \\
\text { FLOODPLAIN } \\
\text { TAPS A/G; LWC }\end{array}$ & $\begin{array}{l}\text { AC?BB? } \\
\text { CN?GR; } \\
\text { LT?RW? }\end{array}$ & JafemrapSSSSSSSSSSSSnode & $\begin{array}{l}829563 \\
873550 \\
681+00 \\
242+00\end{array}$ & $\begin{array}{l}\mathrm{U} \\
14 \mathrm{~S} \\
12 \mathrm{E} \\
07,17 \\
20 \& 32\end{array}$ & $\begin{array}{l}\text { A } \\
\text { B } \\
\text { D } \\
\text { E } \\
\text { FG }\end{array}$ \\
\hline $\begin{array}{c}170.60 \\
170.79 \\
109\end{array}$ & $\begin{array}{l}\text { (West Fork, North Fork, } \\
\text { Chandalar River FLPLN) } \\
\text { TAPS B/G; LWC }\end{array}$ & $\begin{array}{l}\text { CN;CI? } \\
\text { DV?GR; } \\
\text { HW?NP? } \\
\text { RW }\end{array}$ & jafemrapCCCCSSSSCCCCnode & $\begin{array}{r}900767 \\
901746 \\
1093+00 \\
1083+21 \\
\end{array}$ & $\begin{array}{l}\mathrm{U} \\
16 \mathrm{~S} \\
11 \mathrm{E} \\
03\end{array}$ & $\begin{array}{l}\text { AB DE } \\
\text { FG }\end{array}$ \\
\hline $\begin{array}{r}173.28 \\
173.44 \\
109 \\
\end{array}$ & $\begin{array}{l}\text { (West Fork, North Fork, } \\
\text { Chandalar River) } \\
\text { TAPS B/G; BLOCKPOINT }\end{array}$ & $\begin{array}{l}\text { CN;CI? } \\
\text { DV?GR; } \\
\text { HW?NP? } \\
\text { RW }\end{array}$ & jafemrapCCCCSSSSCCCCnode & $\begin{array}{l}914900 \\
915740 \\
957+00 \\
945+23 \\
\end{array}$ & $\begin{array}{l}U \\
16 S \\
11 \mathrm{E} \\
16 \\
\end{array}$ & $\begin{array}{ll}\mathrm{AB} & \mathrm{DE} \\
\mathrm{FG} & \end{array}$ \\
\hline $\begin{array}{c}175.38 \\
109\end{array}$ & $\begin{array}{l}\text { (Andy's CK) } \\
\text { TAPS B/G; LWC }\end{array}$ & $\begin{array}{l}\text { CN;DV; } \\
\text { GR }\end{array}$ & jafemrapSSSSSSSSSSSSnode & $\begin{array}{r}926000 \\
841+65 \\
840+52 \\
\end{array}$ & $\begin{array}{l}\mathrm{U} \\
16 \mathrm{~S} \\
11 \mathrm{E} \\
20 \\
\end{array}$ & $\begin{array}{ll}\mathrm{AB} & \mathrm{E} \\
\mathrm{FG} & \end{array}$ \\
\hline $\begin{array}{c}176.11 \\
109\end{array}$ & $\begin{array}{l}\text { One-Seven-Six Mile Creek } \\
\text { TAPS B/G; LWC }\end{array}$ & $\overline{\mathrm{GR}}$ & jafemrapSSSSSSSSSSSSnode & $\begin{array}{l}929903 \\
929843\end{array}$ & $\begin{array}{l}\mathrm{U} \\
16 \mathrm{~S} \\
11 \mathrm{E} \\
30 \\
\end{array}$ & $F^{B} \quad$ I J \\
\hline $\begin{array}{c}176.68 \\
109\end{array}$ & $\begin{array}{l}\text { Beaver Brook } \\
\text { TAPS B/G; LWC }\end{array}$ & $\begin{array}{l}\text { DV,CD? } \\
\text { GR? }\end{array}$ & jafemrapSSSSSSSSSSSSnode & & $\begin{array}{l}\mathrm{U} \\
16 \mathrm{~S} \\
11 \mathrm{E} \\
30 \\
\end{array}$ & $\begin{array}{ll}\mathrm{A} \mathrm{B} & \\
\mathrm{F} & \text { I J }\end{array}$ \\
\hline $\begin{array}{c}177.28 \\
177.78 \\
108\end{array}$ & $\begin{array}{l}\text { DIETRICH RIVER } \\
\text { TAPS B/G; BLOCKPOINT }\end{array}$ & $\begin{array}{l}\text { BB?CN; } \\
\text { DV;GR; } \\
\text { LS?RW }\end{array}$ & jafemrapSSSSSSSSSSSSnode & $\begin{array}{l}936025 \\
938700 \\
742+44 \\
714+42 \\
\end{array}$ & $\begin{array}{l}U \\
16 S \\
10 \\
25 \& 36 \\
\end{array}$ & $\begin{array}{ll}\mathrm{AB} & \mathrm{E} \\
\mathrm{F} & \end{array}$ \\
\hline $\begin{array}{l}178.79 \\
108\end{array}$ & $\begin{array}{l}\text { (Oskar's Eddy) } \\
\text { TAPS A/C; LWC }\end{array}$ & DV;GR & jemrapSSSSSSSSSSSSnode & $\begin{array}{l}943968 \\
944038 \\
663+02\end{array}$ & $\begin{array}{l}\mathrm{U} \\
17 \mathrm{~S} \\
10 \mathrm{E} \\
02\end{array}$ & $\begin{array}{l}\mathrm{AB} \\
\mathrm{FG}\end{array}$ \\
\hline $\begin{array}{l}179.33 \\
180.41 \\
108\end{array}$ & $\begin{array}{l}\text { DIETRICH RIVER } \\
\text { (Sehroeder's Spring) } \\
\text { TAPS B/C; BLOCKPOINT }\end{array}$ & $\begin{array}{l}\mathrm{BB} ; \mathrm{CN} \\
\mathrm{DV} ; \mathrm{GR} \\
\mathrm{LS} ; \mathrm{RW}\end{array}$ & $\begin{array}{l}\text { CCCCCCCCCCSSSSSSCCCCCCCC } \\
\text { OVERWINTERING AREA } \\
\text { FOR DV; GR; RW }\end{array}$ & $\begin{array}{l}946870 \\
952575 \\
626+00 \\
578+00\end{array}$ & $\begin{array}{l}\mathrm{F} \\
37 \mathrm{~N} \\
09 \mathrm{~W} \\
25 \& \\
35\end{array}$ & $\begin{array}{ll}\mathrm{AB} & \mathrm{E} \\
\mathrm{FG} & \end{array}$ \\
\hline $\begin{array}{c}180.74 \\
181.39 \\
108\end{array}$ & $\begin{array}{l}\text { DIETRICH RIVER } \\
\text { TAPS B/G; BLOCKPOINT }\end{array}$ & $\begin{array}{l}\text { BB;CN; } \\
\text { DV;GR; } \\
\text { LL;RW }\end{array}$ & CCCCCCCCCCSSSSSSCCCCCCCC & $\begin{array}{l}954290 \\
957750 \\
556+00 \\
525+75 \\
\end{array}$ & $\begin{array}{l}\mathrm{F} \\
36 \mathrm{~N} \\
10 \mathrm{~W} \\
02 \& 03 \\
\end{array}$ & $\begin{array}{l}\mathrm{AB} \\
\mathrm{FG}\end{array}$ \\
\hline $\begin{array}{l}181.63 \\
181.93 \\
108\end{array}$ & $\begin{array}{l}\text { DIETRICH RIVER } \\
\text { TAPS B/G; BLOCKPOINT }\end{array}$ & $\begin{array}{l}\text { BB;CN; } \\
\text { DV;GR; } \\
\text { LS;RW }\end{array}$ & CCCCCCCCCCSSSSSSCCCCCCCC & $\begin{array}{l}959000 \\
960600 \\
513+95 \\
500+36 \\
\end{array}$ & $\begin{array}{l}\mathrm{F} \\
36 \mathrm{~N} \\
10 \mathrm{~W} \\
03 \\
\end{array}$ & $\begin{array}{l}\mathrm{AB} \\
\mathrm{FG}\end{array}$ \\
\hline $\begin{array}{c}182.58 \\
184.21 \\
107\end{array}$ & $\begin{array}{l}\text { DIETRICH RIVER } \\
\text { TAPS B/G; BLOCKPOINT }\end{array}$ & $\begin{array}{l}\mathrm{BB} ; \mathrm{CN} \\
\mathrm{DV} ; \mathrm{GR} ; \\
\mathrm{LS} ; \mathrm{RW}\end{array}$ & CCCCCCCCCCSSSSSSCCCCCCCC & $\begin{array}{l}964000 \\
972650 \\
457+37 \\
379+39 \\
\end{array}$ & $\begin{array}{l}\mathrm{F} \\
36 \mathrm{~N} \\
10 \mathrm{~W} \\
10,15,16 \&\end{array}$ & $\begin{array}{l}\mathrm{AB} \\
\mathrm{FG}\end{array}$ \\
\hline
\end{tabular}


A\&G STIPULATION 2.5.3 ZONES OF RESTRICTED ACTIVITIES - KEY FISH AREAS 07/30/03 PAGE 6 of 21

\begin{tabular}{|c|c|c|c|c|c|c|}
\hline $\mathrm{MP}$ & $\begin{array}{l}\text { : STREAM NAME(s) } \\
: \text { Comments }\end{array}$ & $\begin{array}{l}\text { FISH } \\
\text { SPECIES }\end{array}$ & $\begin{array}{l}\text { A: JaFeMrApMaJuJlAuSeOcNoDe } \\
\text { D : PERIOD OF SENSITIVITY } \\
\text { A : } \\
\text { D : }\end{array}$ & $\begin{array}{l}\text { FIELD } \\
: \text { STATION } \\
: \quad \text { G-5 } \\
\end{array}$ & $\begin{array}{l}: \text { MER } \\
: T \\
: \text { R } \\
: \text { SEC }\end{array}$ & $\begin{array}{l}\text { REFER } \\
\text {-ENCE }\end{array}$ \\
\hline & 7 & & $\vdots$ & 7 & 21 & \\
\hline $\begin{array}{c}184.28 \\
107\end{array}$ & $\begin{array}{l}\text { NUTIRWIK CREEK } \\
\text { TAPS B/G; BLOCKPOINT }\end{array}$ & $\begin{array}{l}\mathrm{CN} ; \mathrm{DV} \\
\mathrm{GR} ; \mathrm{RW} ?\end{array}$ & jafemrapSSSSSSSSSSSSnode & $\begin{array}{l}972650 \\
973300 \\
375+54\end{array}$ & $\begin{array}{l}F \\
36 \mathrm{~N} \\
10 \mathrm{~W} \\
21\end{array}$ & $\begin{array}{ll}\mathrm{AB} & \mathrm{E} \\
\mathrm{FG} & \end{array}$ \\
\hline $\begin{array}{c}185.98 \\
107\end{array}$ & $\begin{array}{l}\text { (Beaver Dam Brook) } \\
\text { TAPS B/G; CMP }\end{array}$ & GR? & jafemrapSSSSSSSSSSSSnode & $\begin{array}{l}977750 \\
334+05\end{array}$ & $\begin{array}{l}\mathrm{F} \\
36 \mathrm{~N} \\
10 \mathrm{~W} \\
28\end{array}$ & $\mathbf{F}$ \\
\hline $\begin{array}{c}185.98 \\
186.70 \\
107\end{array}$ & $\begin{array}{l}\text { DIETRICH RIVER } \\
\text { TAPS B/G; BLOCKPOINT }\end{array}$ & $\begin{array}{l}\text { BB;CN; } \\
\text { DV;GR; } \\
\text { LS }\end{array}$ & CCCCCCCCCCSSSSSSCCCCCCCC & $\begin{array}{l}982000 \\
985750 \\
284+64 \\
282+68 \\
280+70 \\
278+94 \\
276+00 \\
259+21 \\
249+14 \\
248+79 \\
\end{array}$ & $\begin{array}{l}\mathrm{F} \\
36 \mathrm{~N} \\
10 \mathrm{~W} \\
28 \& \\
33\end{array}$ & $\begin{array}{cl}\text { B } & \text { E } \\
\text { B } & \text { EF } \\
\text { B } & \text { EF } \\
\text { A } & \text { EF } \\
\text { B } & \text { EF } \\
\text { B } & \text { EF } \\
\text { B } & \text { EF } \\
\text { A } & \text { EF }\end{array}$ \\
\hline $\begin{array}{c}189.92 \\
106\end{array}$ & $\begin{array}{l}\text { (Burger's Bayou) } \\
\text { TAPS B/G; LWC }\end{array}$ & $\begin{array}{l}\mathrm{BB} ; \mathrm{CN} \\
\mathrm{DV} ; \mathrm{GR}\end{array}$ & jafemrapSSSSSSSSSSSSnode & $\begin{array}{r}1002800 \\
72+43\end{array}$ & $\begin{array}{l}F \\
35 \mathrm{~N} \\
10 \mathrm{~W} \\
16\end{array}$ & $\begin{array}{l}\mathrm{AB} \\
\mathrm{F}\end{array}$ \\
\hline $\begin{array}{c}192.33 \\
192.99 \\
106\end{array}$ & $\begin{array}{l}\text { DIETRICH RIVER (Big Jim } \\
\text { Overwintering Area) } \\
\text { TAPS B/G; BLOCKPOINT }\end{array}$ & $\begin{array}{l}\text { BB?CN } \\
\text { DV?GR; } \\
\text { LS?RW }\end{array}$ & $\begin{array}{l}\text { CCCCCCCCCCSSSSSSCCCCCCCC } \\
\text { BIG JIM OVERWINTERING AREA }\end{array}$ & $\begin{array}{l}1015500 \\
1019000 \\
2195+05 \\
2182+00\end{array}$ & $\begin{array}{l}\mathrm{F} \\
35 \mathrm{~N} \\
10 \mathrm{~W} \\
33\end{array}$ & $\begin{array}{l}\mathrm{AB} \\
\mathrm{FG}\end{array}$ \\
\hline $\begin{array}{c}194.04 \\
195.91 \\
105\end{array}$ & $\begin{array}{l}\text { DIETRICH RIVER } \\
\text { TAPS B/G; BLOCKPOINT }\end{array}$ & $\begin{array}{l}\text { BB?CN; } \\
\text { DV;GR; } \\
\text { LS?RW }\end{array}$ & $\begin{array}{l}\text { jafemrapCCCCSSSSCCCCnode } \\
\text { (includes Ugh Creek which passes } \\
\text { through CMP in dike) }\end{array}$ & $\begin{array}{l}1024536 \\
1034400 \\
2108+00 \\
2011+33\end{array}$ & $\begin{array}{l}\mathrm{F} \\
34 \mathrm{~N} \\
10 \mathrm{~W} \\
04,09 \\
10 \& 15\end{array}$ & $\begin{array}{ll}\mathrm{AB} & \mathrm{E} \\
\mathrm{F} & \mathrm{I}\end{array}$ \\
\hline $\begin{array}{c}196.98 \\
105\end{array}$ & $\begin{array}{l}\text { (Stanzla CK) } \\
\text { TAPS A/G; LWC }\end{array}$ & $\begin{array}{l}\text { GR;LS; } \\
\text { RW }\end{array}$ & jafemrapCCCCSSSSCCCCnode & $\begin{array}{l}1040054 \\
1040107 \\
1952+70\end{array}$ & $\begin{array}{l}F \\
34 N \\
10 W \\
22\end{array}$ & $\begin{array}{ll}\mathrm{AB} & \mathrm{E} \\
\mathrm{F} & \end{array}$ \\
\hline $\begin{array}{c}197.08 \\
105\end{array}$ & $\begin{array}{l}\text { (Dunder's Dribble) } \\
\text { TAPS AVG; LWC }\end{array}$ & $\begin{array}{l}\mathrm{CN} ; \mathrm{GR} ; \\
\mathrm{RW} \text { ? }\end{array}$ & SSSSSSSSSSSSSSSSSSSSSSSS & $\begin{array}{l}1040534 \\
1040589 \\
1947+76\end{array}$ & $\begin{array}{l}\mathrm{F} \\
34 \mathrm{~N} \\
10 \mathrm{~W} \\
22 \\
\end{array}$ & $\begin{array}{l}\mathrm{AB} \\
\mathrm{F}\end{array}$ \\
\hline $\begin{array}{c}197.19 \\
105\end{array}$ & $\begin{array}{l}\text { (Number Lakes CK) } \\
\text { TAPS A/G; LWC }\end{array}$ & $\mathrm{CN} ; \mathrm{GR}$ & jafemrapCCCCSSCCCCCCnode & $\begin{array}{l}1041136 \\
1041207 \\
1940+81\end{array}$ & $\begin{array}{l}\mathrm{F} \\
34 \mathrm{~N} \\
10 \mathrm{~W} \\
22\end{array}$ & $\begin{array}{l}\mathrm{AB} \\
\mathrm{F}\end{array}$ \\
\hline $\begin{array}{c}197.86 \\
105\end{array}$ & $\begin{array}{l}\text { (Snowden Pond Outlet) } \\
\text { TAPS A/G; LWC }\end{array}$ & $\mathrm{CN} ; \mathrm{GR} ?$ & jafemrapSSSSSSSSSSSSnode & $\begin{array}{l}1044680 \\
1044740 \\
1906+65\end{array}$ & $\begin{array}{l}\mathrm{F} \\
34 \mathrm{~N} \\
10 \mathrm{~W} \\
27\end{array}$ & $\begin{array}{l}\mathrm{AB} \\
\mathrm{F}\end{array}$ \\
\hline $\begin{array}{c}198.56 \\
105\end{array}$ & $\begin{array}{l}\text { SNOWDEN CREEK } \\
\text { TAPS A/G; LWC }\end{array}$ & $\begin{array}{l}\mathrm{CN} ; \mathrm{DV} ; \\
\mathrm{GR} ; \mathrm{RW} ?\end{array}$ & jafemrapSSSSSSSSSSSSnode & $\begin{array}{l}1048364 \\
1048432 \\
1869+54\end{array}$ & $\begin{array}{l}F \\
34 \mathrm{~N} \\
10 \mathrm{~W} \\
26\end{array}$ & $\begin{array}{l}\mathrm{AB} \\
\mathrm{FG}\end{array}$ \\
\hline $\begin{array}{c}200.03 \\
104\end{array}$ & $\begin{array}{l}\text { (Sahr's Slough) } \\
\text { TAPS A/G; CMP }\end{array}$ & GR & jafemrapSSSSSSauseocnode & $\begin{array}{l}1056107 \\
1056171 \\
1794+00\end{array}$ & $\begin{array}{l}\mathrm{F} \\
33 \mathrm{~N} \\
10 \mathrm{~W} \\
02 \\
\end{array}$ & $\begin{array}{l}\mathrm{AB} \\
\mathrm{F}\end{array}$ \\
\hline 200.17 & $\begin{array}{l}\text { (Sahr's Slough) } \\
\text { MP 200 B YPASS }\end{array}$ & GR & jafemrapSSSSSSauseocnode & $\begin{array}{l}1056908 \\
1056970\end{array}$ & $\begin{array}{ll}\mathrm{F} \\
33 \mathrm{~N}\end{array}$ & \\
\hline
\end{tabular}


A\&G STIPULATION 2.5.3 ZONES OF RESTRICTED ACTIVITIES - KEY FISH AREAS $\quad$ 07/30/03 PAGE 7 of 21

\begin{tabular}{|c|c|c|c|c|c|c|}
\hline MP & STREAM NAME(s) & $\begin{array}{l}\text { FISH } \\
\text { SPECIES }\end{array}$ & $\begin{array}{l}\text { A: JaFeMrApMaJuJlAuSeOcNoDe } \\
\text { D : PERIOD OF SENSITIVITY }\end{array}$ & $\begin{array}{l}\text { FIELD } \\
\text { STATION }\end{array}$ & MER & $\begin{array}{l}\text { REFER } \\
\text {-ENCE }\end{array}$ \\
\hline $\mathrm{A} / \mathrm{S}$ & Comments & : & $\begin{array}{l}\text { A : } \\
\text { D : }\end{array}$ & G-5 & REC & \\
\hline 104 & TAPS A/G; CMP & & & & $\begin{array}{l}10 \mathrm{~W} \\
02\end{array}$ & \\
\hline $\begin{array}{c}200.16 \\
200.72 \\
104\end{array}$ & $\begin{array}{l}\text { DIETRICH RIVER } \\
\text { SECTION BYPASSED AT } \\
\text { MP } 200 \text { REPAIR } \\
\text { TAPS B/G; BLOCKPOINT }\end{array}$ & $\begin{array}{l}\text { BB;CN; } \\
\text { DV;GR; } \\
\text { LS;RW }\end{array}$ & CCCCCCCCCCSSSSSSCCCCCCCC & $\begin{array}{l}1056833 \\
1059782 \\
1785+60 \\
1756+00 \\
\end{array}$ & $\begin{array}{l}\mathrm{F} \\
33 \mathrm{~N} \\
10 \mathrm{~W} \\
02\end{array}$ & $\begin{array}{l}\mathrm{AB} \\
\mathrm{F}\end{array}$ \\
\hline $\begin{array}{c}201.43 \\
104\end{array}$ & $\begin{array}{l}\text { (Disaster CK) } \\
\text { TAPS A/G; LWC }\end{array}$ & $\overline{\mathrm{CN}} ; \mathrm{GR}$ & jafemrapSSSSSSSSSSSSnode & $\begin{array}{l}1063529 \\
1063603 \\
1718+00\end{array}$ & $\begin{array}{l}\mathrm{F} \\
33 \mathrm{~N} \\
10 \mathrm{~W} \\
11\end{array}$ & $\begin{array}{l}\mathrm{AB} \\
\mathrm{FG}\end{array}$ \\
\hline $\begin{array}{c}202.15 \\
104\end{array}$ & $\begin{array}{l}\text { (Airport CK) } \\
\text { TAPS A/G; LWC }\end{array}$ & DV;GR & jafemrapSSSSSSSSSSSSnode & $\begin{array}{l}1067293 \\
1067359 \\
1680+00\end{array}$ & $\begin{array}{l}\mathrm{F} \\
33 \mathrm{~N} \\
10 \mathrm{~W} \\
14 \\
\end{array}$ & $\begin{array}{l}\mathrm{AB} \\
\mathrm{F}\end{array}$ \\
\hline $\begin{array}{c}203.64 \\
104\end{array}$ & $\begin{array}{l}\text { (Steitz Lake Outlet) } \\
\text { TAPS A/G; LWC }\end{array}$ & $\begin{array}{l}\text { BB;CN; } \\
\text { DS?DV? } \\
\text { GR;LS }\end{array}$ & jafemrapCCCCSSSSCCCCnode & $\begin{array}{l}1075159 \\
1075229 \\
1608+00\end{array}$ & $\begin{array}{l}\mathrm{F} \\
33 \mathrm{~N} \\
10 \mathrm{~W} \\
24 \\
\end{array}$ & $\begin{array}{l}\mathrm{AB} \\
\mathrm{FG}\end{array}$ \\
\hline $\begin{array}{c}204.08 \\
104\end{array}$ & $\begin{array}{l}\text { (Brockman CK) } \\
\text { TAPS A/G; SUMMER } \\
\text { BLOCKPOINT }\end{array}$ & $\begin{array}{l}\text { CN;DV; } \\
\text { GR;RW }\end{array}$ & jafemrapSSSSSSSSSSSSnode & $\begin{array}{l}1077385 \\
1077679 \\
1581+65 \\
1579+18 \\
\end{array}$ & $\begin{array}{l}\mathrm{F} \\
33 \mathrm{~N} \\
10 \mathrm{~W} \\
25\end{array}$ & $\begin{array}{l}\mathrm{AB} \\
\mathrm{FG}\end{array}$ \\
\hline 205.34 & $\begin{array}{l}\text { DIETRICH RIVER } \\
\text { TAPS A/G; BLOCKPOINT }\end{array}$ & $\begin{array}{l}\text { BB;CN; } \\
\text { DV?GR; } \\
\text { KS?LS } \\
\text { RW }\end{array}$ & $\begin{array}{c}\text { CCCCCCCCCCSSSSSSCCCCCCCC } \\
.\end{array}$ & $\begin{array}{l}1084002 \\
1084402 \\
1533+95 \\
1526+55\end{array}$ & $\begin{array}{l}\mathrm{F} \\
33 \mathrm{~N} \\
10 \mathrm{~W} \\
35\end{array}$ & $\begin{array}{l}\mathrm{AB} \\
\mathrm{F}\end{array}$ \\
\hline $\begin{array}{c}205.74 \\
103\end{array}$ & $\begin{array}{l}\text { (Eva's Alv) } \\
\text { TAPS A/G; LWC }\end{array}$ & $\begin{array}{l}\text { CD;GR } \\
\text { DV }\end{array}$ & jafemrSSSSSSSSSSSSSSnode & $\begin{array}{l}1086260 \\
1086330 \\
1507+55\end{array}$ & $\begin{array}{l}\mathrm{F} \\
33 \mathrm{~N} \\
10 \mathrm{~W} \\
35\end{array}$ & $\begin{array}{l}\mathrm{AB} \\
\mathrm{F}\end{array}$ \\
\hline $\begin{array}{c}208.01 \\
208.45 \\
103\end{array}$ & $\begin{array}{l}\text { Middle Fork KOYUKUK } \\
\text { RIVER } \\
\text { TAPS A/G; BLOCKPOINT }\end{array}$ & $\begin{array}{l}\text { BB?CN; } \\
\text { DS?DV; } \\
\text { GR;LS; } \\
\text { NP?RW }\end{array}$ & 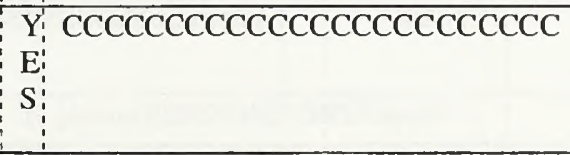 & $\begin{array}{l}1098300 \\
1100600 \\
1384+47 \\
1361+45 \\
\end{array}$ & $\begin{array}{l}F \\
32 \\
10 W \\
09\end{array}$ & $\begin{array}{l}\mathrm{AB} \\
\mathrm{F}\end{array}$ \\
\hline $\begin{array}{c}209.02 \\
103\end{array}$ & $\begin{array}{l}\text { (North Fork, Sukakpak CK) } \\
\text { TAPS A/G; LWC }\end{array}$ & $\mathrm{CD} ; \mathrm{GR}$ & jafemrSSSSSSSSSSSSSSnode & $\begin{array}{l}1103583 \\
1103643 \\
1332+30\end{array}$ & $\begin{array}{l}\mathrm{F} \\
32 \mathrm{~N} \\
10 \mathrm{~W} \\
16\end{array}$ & BC \\
\hline $\begin{array}{c}209.54 \\
103\end{array}$ & $\begin{array}{l}\text { (West Fork, Sukapak CK or } \\
\text { Pamplin's Potholes) } \\
\text { TAPS A/G; LWC }\end{array}$ & $\begin{array}{l}\text { CN;DV; } \\
\text { GR }\end{array}$ & jafemrCCCCCCSSSSCCCCnode & $\begin{array}{l}1106337 \\
1106407 \\
1305+50\end{array}$ & $\begin{array}{l}\mathrm{F} \\
32 \mathrm{~N} \\
10 \mathrm{~W} \\
16\end{array}$ & $\begin{array}{l}\text { A B C D } \\
\text { F }\end{array}$ \\
\hline $\begin{array}{c}210.22 \\
103\end{array}$ & $\begin{array}{l}\text { (Marsh CK) } \\
\text { TAPS A/G; CMP }\end{array}$ & $\mathrm{CN} ; \mathrm{GR}$ & jafemrCCCCCCSSSSCCCCnode & $\begin{array}{l}1109943 \\
1110003 \\
1268+96\end{array}$ & $\begin{array}{l}F \\
32 \mathrm{~N} \\
10 \mathrm{~W} \\
21 \\
\end{array}$ & $\begin{array}{l}\text { A B C } \\
\text { F }\end{array}$ \\
\hline $\begin{array}{c}210.43 \\
103\end{array}$ & $\begin{array}{l}\text { (Marsh CK) } \\
\text { Fish parallel to ROW } \\
\text { TAPS A/G; LWC }\end{array}$ & $\mathrm{CN} ; \mathrm{GR}$ & jafemrCCCCCCSSSSCCCCnode & $\begin{array}{l}1111082 \\
1111022\end{array}$ & $\begin{array}{l}\mathrm{F} \\
32 \mathrm{~N} \\
10 \mathrm{~W} \\
21 \\
\end{array}$ & J \\
\hline
\end{tabular}


A\&G STIPULATION 2.5.3 ZONES OF RESTRICTED ACTIVITIES - KEY FISH AREAS

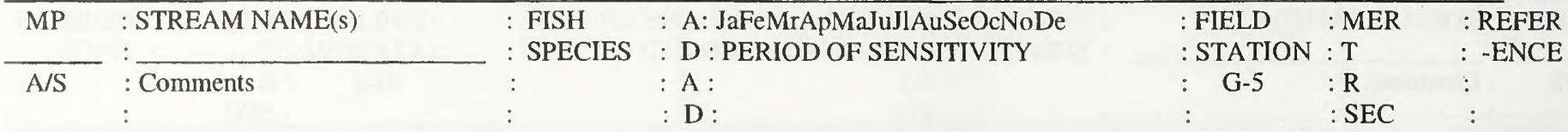

\begin{tabular}{|c|c|c|c|c|c|c|}
\hline $\begin{array}{c}210.57 \\
103\end{array}$ & $\begin{array}{l}\text { (Marsh CK) } \\
\text { TAPS A/G; LWC }\end{array}$ & $\begin{array}{l}\text { CN;GR } \\
\text { LS }\end{array}$ & jafemrCCCCCCSSSSCCCCnode & $\begin{array}{l}1111792 \\
1111862 \\
1258+35\end{array}$ & $\begin{array}{l}\mathrm{F} \\
32 \mathrm{~N} \\
10 \mathrm{~W} \\
21\end{array}$ & $\begin{array}{l}\text { A B C } \\
F\end{array}$ \\
\hline $\begin{array}{c}210.94 \\
103\end{array}$ & $\begin{array}{l}\text { (Marsh CK) } \\
\text { TAPS B/G; LWC }\end{array}$ & $\mathrm{CN} ; \mathrm{GR}$ & jafemrCCCCCCSSSSCCCCnode & $\begin{array}{l}1113750 \\
1250+00\end{array}$ & $\begin{array}{l}\mathrm{F} \\
32 \mathrm{~N} \\
10 \mathrm{~W} \\
20\end{array}$ & $\begin{array}{l}\mathrm{ABC} \\
\mathrm{F}\end{array}$ \\
\hline $\begin{array}{c}210.94 \\
211.41 \\
103\end{array}$ & $\begin{array}{l}\text { Middle Fork, KOYUKUK } \\
\text { RIVER } \\
\text { TAPS B/G; BLOCKPOINT }\end{array}$ & $\begin{array}{l}\text { BB? CN } \\
\text { DS?DV; } \\
\text { GR;LS; } \\
\text { NP?RW }\end{array}$ & \begin{tabular}{l:l}
$Y$ SSSSSSCCCCCCSSSSCCCCSSSS \\
\hdashline
\end{tabular} & $\begin{array}{l}1113750 \\
1116250 \\
1237+55 \\
1207+63\end{array}$ & $\begin{array}{l}\mathrm{F} \\
32 \mathrm{~N} \\
10 \mathrm{~W} \\
20,29\end{array}$ & $\begin{array}{l}\text { A B } \\
\text { F }\end{array}$ \\
\hline $\begin{array}{r}215.31 \\
102\end{array}$ & $\begin{array}{l}\text { LINDA CREEK } \\
\text { TAPS A/G; BLOCKPOINT }\end{array}$ & $\mathrm{CN} ; \mathrm{GR}$ & jafemrSSSSSSSSSSSSSSnode & $\begin{array}{l}1136782 \\
1136900 \\
1001+18\end{array}$ & $\begin{array}{l}\mathrm{F} \\
31 \mathrm{~N} \\
10 \mathrm{~W} \\
07\end{array}$ & $\begin{array}{l}\text { A B } \\
\text { F G }\end{array}$ \\
\hline $\begin{array}{r}215.81 \\
102\end{array}$ & $\begin{array}{l}\text { GOLD CREEK } \\
\text { TAPS B/G; SUMMER } \\
\text { BLOCKPOINT }\end{array}$ & $\begin{array}{l}\text { CN;DV? } \\
\text { GR;RW? }\end{array}$ & jafemrCCCCCCSSSSCCCCnode & $\begin{array}{r}1139410 \\
1139550 \\
976+00\end{array}$ & $\begin{array}{l}\text { F } \\
31 \mathrm{~N} \\
10 \mathrm{~W} \\
18\end{array}$ & $\begin{array}{l}\text { A B } \\
\text { F G }\end{array}$ \\
\hline $\begin{array}{c}216.30 \\
102\end{array}$ & $\begin{array}{l}\text { (Cushing CK) } \\
\text { TAPS A/G; SUMMER } \\
\text { BLOCKPOINT }\end{array}$ & GR? & jafemrSSSSSSSSSSSSSSnode & $\begin{array}{r}1142019 \\
1142074 \\
948+66\end{array}$ & $\begin{array}{l}\mathrm{F} \\
31 \mathrm{~N} \\
10 \mathrm{~W} \\
18\end{array}$ & $\mathrm{E}$ \\
\hline $\begin{array}{c}216.59 \\
102\end{array}$ & $\begin{array}{l}\text { SHEEP CREEK } \\
\text { TAPS A/G; LWC }\end{array}$ & $\mathrm{CN} ; \mathrm{GR}$ & jafemrCCCCCCSSSSCCCCnode & $\begin{array}{r}1143574 \\
1143369 \\
933+01\end{array}$ & $\begin{array}{l}\mathrm{F} \\
31 \mathrm{~N} \\
10 \mathrm{~W} \\
19 \\
\end{array}$ & $\begin{array}{l}\text { A B } \\
\text { F }\end{array}$ \\
\hline $\begin{array}{c}217.09 \\
102\end{array}$ & $\begin{array}{l}\text { WOLF PUP CREEK } \\
\text { TAPS A/G; CMP }\end{array}$ & $\mathrm{CN} ; \mathrm{GR} ?$ & jafemrSSSSSSSSSSSSSSnode & $\begin{array}{r}1146220 \\
1146269 \\
906+78\end{array}$ & $\begin{array}{l}\mathrm{F} \\
31 \mathrm{~N} \\
10 \mathrm{~W} \\
19\end{array}$ & $\begin{array}{l}\mathrm{AB} \\
\mathrm{F}\end{array}$ \\
\hline $\begin{array}{c}218.00 \\
101\end{array}$ & $\begin{array}{l}\text { (Alignment Slough) } \\
\text { TAPS A/G; CMP }\end{array}$ & CD? GR & jafemrCCCCCCSSSSCCCCnode & $\begin{array}{r}1150992 \\
1151052 \\
859+99\end{array}$ & $\begin{array}{l}\mathrm{F} \\
31 \mathrm{~N} \\
11 \mathrm{~W} \\
25 \\
\end{array}$ & $\begin{array}{l}\mathrm{AB} \\
\mathrm{F}\end{array}$ \\
\hline $\begin{array}{c}218.08 \\
101\end{array}$ & $\begin{array}{l}\text { (Alignment Slough) } \\
\text { TAPS A/G; CMP }\end{array}$ & CD? GR & jafemrCCCCCCSSSSCCCCnode & $\begin{array}{r}1151409 \\
1151469 \\
855+94\end{array}$ & $\begin{array}{l}\mathrm{F} \\
31 \mathrm{~N} \\
11 \mathrm{~W} \\
25\end{array}$ & $\begin{array}{l}\text { A B } \\
\text { F }\end{array}$ \\
\hline $\begin{array}{c}218.20 \\
101\end{array}$ & $\begin{array}{l}\text { (Alignment Slough) } \\
\text { TAPS A/G; CMP }\end{array}$ & CD? GR & jafemrCCCCCCSSSSCCCCnode & $\begin{array}{r}1152070 \\
1152139 \\
845+28\end{array}$ & $\begin{array}{l}\mathrm{F} \\
31 \mathrm{~N} \\
11 \mathrm{~W} \\
25\end{array}$ & $\begin{array}{l}\text { A B } \\
\text { F }\end{array}$ \\
\hline $\begin{array}{c}218.35 \\
101\end{array}$ & $\begin{array}{l}\text { (Alignment Slough) } \\
\text { TAPS A/G; CMP }\end{array}$ & CD? GR & jafemrCCCCCCSSSSCCCCnode & $\begin{array}{r}1152847 \\
1152902 \\
840+20\end{array}$ & $\begin{array}{l}\mathrm{F} \\
31 \mathrm{~N} \\
11 \mathrm{~W} \\
25\end{array}$ & $\begin{array}{l}\text { A B } \\
\text { F }\end{array}$ \\
\hline $\begin{array}{c}218.45 \\
101\end{array}$ & $\begin{array}{l}\text { (Alignment Slough) } \\
\text { TAPS A/G; CMP }\end{array}$ & CD? GR & jafemrCCCCCCSSSSCCCCnode & $\begin{array}{r}1153361 \\
1153434 \\
835+63\end{array}$ & $\begin{array}{l}\mathrm{F} \\
31 \mathrm{~N} \\
11 \mathrm{~W} \\
25\end{array}$ & $\begin{array}{l}\text { A B } \\
F\end{array}$ \\
\hline
\end{tabular}


A\&G STIPULATION 2.5.3 ZONES OF RESTRICTED ACTIVITIES - KEY FISH AREAS 07/30/03 PAGE 9 of 21

\begin{tabular}{|c|c|c|c|c|c|c|}
\hline $\begin{array}{l}\mathrm{MP} \\
\mathrm{A} / \mathrm{S}\end{array}$ & $\begin{array}{l}: \text { STREAM NAME(s) } \\
: \text { Comments }\end{array}$ & $\begin{array}{l}\text { FISH } \\
: \text { SPECIES } \\
:\end{array}$ & $\begin{array}{l}\text { A: JaFeMrApMaJuJlAuSeOcNoDe } \\
\text { : D : PERIOD OF SENSITIVITY } \\
\text { A : } \\
\text { : D : }\end{array}$ & $\begin{array}{l}\text { :FIELD } \\
: \text { STATION } \\
: \quad \text { G-5 }\end{array}$ & $\begin{array}{l}\text { MER } \\
: \mathrm{T} \\
: \mathrm{R} \\
\mathrm{SEC}\end{array}$ & $\begin{array}{l}\text { : REFER } \\
: \text {-ENCE } \\
: \\
:\end{array}$ \\
\hline $\begin{array}{c}219.04 \\
101\end{array}$ & $\begin{array}{l}\text { OVER CREEK (Complex) } \\
\text { TAPS A/G; CMP }\end{array}$ & BB;GR & $\begin{array}{l}\text { jafemrSSSSSSSSSSSSSSnode } \\
\text { jasS }\end{array}$ & $\begin{array}{r}1156490 \\
1156552 \\
805+39\end{array}$ & $\begin{array}{l}\mathrm{F} \\
31 \mathrm{~N} \\
11 \mathrm{~W} \\
26\end{array}$ & $\begin{array}{l}\mathrm{AB} \\
\mathrm{F}\end{array}$ \\
\hline 219.08 & $\begin{array}{l}\text { Tributary OVER CREEK } \\
\text { TAPS A/G; LWC }\end{array}$ & BB; GR & jafemrSSSSSSSSSSSSSSnode & $\begin{array}{r}1156732 \\
1156793 \\
803+12\end{array}$ & $\begin{array}{l}\mathrm{F} \\
31 \mathrm{~N} \\
11 \mathrm{~W} \\
26\end{array}$ & $\begin{array}{l}\mathrm{AB} \\
\mathrm{F}\end{array}$ \\
\hline $\begin{array}{c}219.13 \\
101\end{array}$ & $\begin{array}{l}\text { OVER CREEK (Complex) } \\
\text { TAPS A/G; CMP }\end{array}$ & BB; GR & jafemrSSSSSSSSSSSSSSnode & $\begin{array}{r}1156963 \\
1157033 \\
802+12\end{array}$ & $\begin{array}{l}\mathrm{F} \\
31 \mathrm{~N} \\
11 \mathrm{~W} \\
35\end{array}$ & $\begin{array}{l}\text { A B } \\
F\end{array}$ \\
\hline $\begin{array}{c}219.20 \\
101\end{array}$ & $\begin{array}{l}\text { OVER CREEK (Complex or } \\
\text { Rainbow CK) } \\
\text { TAPS A/G; CMP }\end{array}$ & $\begin{array}{l}\mathrm{BB} ; \mathrm{GR} \\
\mathrm{CN}\end{array}$ & jafemrSSSSSSSSSSSSSSnode & $\begin{array}{r}1157333 \\
1157403 \\
796+36\end{array}$ & $\begin{array}{l}\mathrm{F} \\
31 \mathrm{~N} \\
11 \mathrm{~W} \\
35\end{array}$ & $\begin{array}{l}\text { A B } \\
\text { F }\end{array}$ \\
\hline $\begin{array}{c}219.52 \\
101\end{array}$ & $\begin{array}{l}\text { (Richardson Slough or Coon } \\
\text { Gulch?) } \\
\text { TAPS A/G; LWC }\end{array}$ & $\begin{array}{l}\text { GR; LS? } \\
\text { RW? }\end{array}$ & jafemrSSSSSSSSSSSSSSnode & $\begin{array}{r}1159070 \\
1159135 \\
781+90\end{array}$ & $\begin{array}{l}\mathrm{F} \\
31 \mathrm{~N} \\
11 \mathrm{~W} \\
35 \\
\end{array}$ & $\mathrm{~F}$ \\
\hline $\begin{array}{r}219.60 \\
101\end{array}$ & $\begin{array}{l}\text { (Richardson Slough) } \\
\text { TAPS A/G; LWC }\end{array}$ & $\begin{array}{l}\text { CN? GR; } \\
\text { LS; RW }\end{array}$ & jafemrSSSSSSSSSSSSSSnode & $\begin{array}{r}1159430 \\
1159495 \\
778+00\end{array}$ & $\begin{array}{l}\mathrm{F} \\
31 \mathrm{~N} \\
11 \mathrm{~W} \\
35 \\
\end{array}$ & $\begin{array}{l}\mathrm{AB} \\
\mathrm{F}\end{array}$ \\
\hline $\begin{array}{r}221.79 \\
101\end{array}$ & $\begin{array}{l}\text { (One-0-One CK) } \\
\text { TAPS A/G; LWC }\end{array}$ & $\begin{array}{l}\text { CN; GR; } \\
\text { LS? }\end{array}$ & jafemrCCCCCCSSSSCCCCnode & $\begin{array}{r}1171039 \\
1171101 \\
664+00\end{array}$ & $\begin{array}{l}\mathrm{F} \\
31 \mathrm{~N} \\
11 \mathrm{~W} \\
33 \\
\end{array}$ & $\begin{array}{l}\text { A B } \\
\text { F }\end{array}$ \\
\hline $\begin{array}{c}221.96 \\
222.07 \\
101\end{array}$ & $\begin{array}{l}\text { Middle Fork, KOYUKUK } \\
\text { RIVER } \\
\text { TAPS A/G; BLOCKPOINT }\end{array}$ & $\begin{array}{l}\text { BB? CN } \\
\text { CS? DS; } \\
\text { DV; GR; } \\
\text { KS?LS; } \\
\text { NP?RW }\end{array}$ & 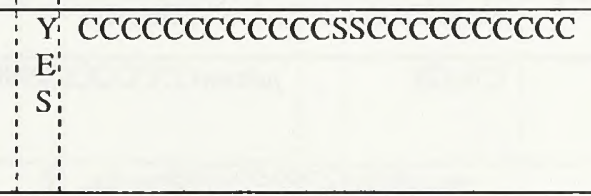 & $\begin{array}{r}1171932 \\
1172525 \\
651+50\end{array}$ & $\begin{array}{l}\mathrm{F} \\
31 \mathrm{~N} \\
11 \mathrm{~W} \\
33\end{array}$ & $\begin{array}{l}\mathrm{AB} \\
\mathrm{F}\end{array}$ \\
\hline $\begin{array}{c}222.28 \\
222,32 \\
101\end{array}$ & $\begin{array}{l}\text { HAMMOND RIVER } \\
\text { TAPS A/G; BLOCKPOINT }\end{array}$ & $\begin{array}{l}\text { CN;DV; } \\
\text { GR;KS } \\
\text { WF;DS } \\
\text { LS }\end{array}$ & $\begin{array}{c:c} & \text { jafemrSSSSSSSSSSSSSSnode } \\
\mathrm{E}^{\prime} & \\
\mathrm{S} & \\
& \end{array}$ & $\begin{array}{r}1173644 \\
1173864 \\
635+50\end{array}$ & $\begin{array}{l}\mathrm{F} \\
31 \mathrm{~N} \\
11 \mathrm{~W} \\
32,33 \\
\end{array}$ & $\begin{array}{l}\mathrm{AB} \\
\mathrm{F}\end{array}$ \\
\hline 224.23 & $\begin{array}{l}\text { (Union Gulch CK) } \\
\text { TAPS A/G; LWC }\end{array}$ & $\begin{array}{l}\text { CN;GR; } \\
\text { LS?RW }\end{array}$ & jafemrCCCCCCSSSSCCCCnode & $\begin{array}{r}1183878 \\
1183948 \\
535+90\end{array}$ & $\begin{array}{l}\mathrm{F} \\
30 \mathrm{~N} \\
11 \mathrm{~W} \\
07\end{array}$ & $\begin{array}{l}\mathrm{AB} \\
\mathrm{F}\end{array}$ \\
\hline $\begin{array}{c}224.55 \\
100\end{array}$ & $\begin{array}{l}\text { (Union Gulch CK) } \\
\text { TAPS A/G; BLOCKPOINT }\end{array}$ & $\begin{array}{l}\text { CN;GR; } \\
\text { LS;RW }\end{array}$ & jafemrCCCCCCSSSSCCCCnode & $\begin{array}{r}1185606 \\
1185666 \\
517+98\end{array}$ & $\begin{array}{l}\mathrm{F} \\
30 \mathrm{~N} \\
11 \mathrm{~W} \\
07 \\
\end{array}$ & A B \\
\hline $\begin{array}{c}224.62 \\
222.94 \\
101\end{array}$ & $\begin{array}{l}\text { Middle Fork, KOYUKUK } \\
\text { RIVER } \\
\text { TAPS A/G; BLOCKPOINT }\end{array}$ & $\begin{array}{l}\text { CN;DS; } \\
\text { DV;GR; } \\
\text { KS;LS; } \\
\text { NP?RW }\end{array}$ & $\begin{array}{l:l} & \text { CCCCCCCCCCCCSSCCCCCCCCCC } \\
E & \\
S & \end{array}$ & $\begin{array}{r}1186000 \\
1187700 \\
517+24 \\
495+50 \\
\end{array}$ & $\begin{array}{l}\mathrm{F} \\
30 \mathrm{~N} \\
11 \mathrm{~W} \\
07\end{array}$ & $\begin{array}{l}\text { A B } \\
F\end{array}$ \\
\hline $\begin{array}{c}225.76 \\
100\end{array}$ & $\begin{array}{l}\text { MINNIE CREEK } \\
\text { TAPS A/G; BLOCKPOINT }\end{array}$ & $\begin{array}{l}\text { BB;CN; } \\
\text { DV;GR; } \\
\text { LS;RW }\end{array}$ & jafemrSSSSSSSSSSSSSSnode & $\begin{array}{r}1191996 \\
1192060 \\
453+95\end{array}$ & $\begin{array}{l}\mathrm{F} \\
30 \mathrm{~N} \\
11 \mathrm{~W} \\
18 \\
\end{array}$ & $\begin{array}{l}\text { A B } \\
\text { F G }\end{array}$ \\
\hline 227.39 & $\begin{array}{l}\text { (Confusion CK) } \\
\text { TAPS B/G; CMP }\end{array}$ & $\mathrm{CN} ; \mathrm{GR}$ & jafemrSSSSSSSSSSSSSSnode & $\begin{array}{r}1200600 \\
1200659 \\
369+00\end{array}$ & $\begin{array}{l}\mathrm{F} \\
30 \mathrm{~N} \\
11 \mathrm{~W} \\
30 \\
\end{array}$ & $\begin{array}{l}\text { A B } \\
\text { F }\end{array}$ \\
\hline
\end{tabular}


A\&G STIPULATION 2.5.3 ZONES OF RESTRICTED ACTIVITIES - KEY FISH AREAS 07/30/03 PAGE 10 of 21

\begin{tabular}{|c|c|c|c|c|c|c|}
\hline MP & : STREAM NAME(s) & : FISH & : A: JaFeMrApMaJuJlAuSeOcNoDe & FIELD & MER & $\begin{array}{l}\text { : REFER } \\
: \text {-ENCE }\end{array}$ \\
\hline $\mathrm{A} / \mathrm{S}$ & : Comments & : & : A : & G-5 & $: \mathrm{R}$ & $:$ \\
\hline
\end{tabular}

\begin{tabular}{|c|c|c|c|c|c|c|}
\hline $\begin{array}{c}232.78 \\
99\end{array}$ & $\begin{array}{l}\text { (Pence's Pond) } \\
\text { TAPS B/G; CMP }\end{array}$ & CD?GR & jafemrSSSSSSSSSSSSSSnode & $\begin{array}{r}1229086 \\
85+80\end{array}$ & $\begin{array}{l}\mathrm{F} \\
29 \mathrm{~N} \\
12 \mathrm{~W} \\
23\end{array}$ & $\begin{array}{ll}\text { A B } & \text { E } \\
\text { F G } & \end{array}$ \\
\hline $\begin{array}{c}233.26 \\
99\end{array}$ & $\begin{array}{l}\text { MARION CREEK } \\
\text { TAPS B/G; BLOCKPOINT }\end{array}$ & $\begin{array}{l}\text { CN;DV; } \\
\text { GR;KS;R } \\
\text { W }\end{array}$ & jafemrSSSSSSSSSSSSSSnode & $\begin{array}{r}1231600 \\
61+85 \\
59+85\end{array}$ & $\begin{array}{l}\mathrm{F} \\
29 \mathrm{~N} \\
12 \mathrm{~W} \\
23\end{array}$ & $\begin{array}{l}\mathrm{A} \mathrm{B} \\
\mathrm{F} \mathrm{G}\end{array}$ \\
\hline $\begin{array}{c}233.38 \\
99\end{array}$ & $\begin{array}{l}\text { (Sharon CK) } \\
\text { TAPS B/G; CMP }\end{array}$ & $\begin{array}{l}\text { BB?CN? } \\
\text { GR?LS? } \\
\text { RW? }\end{array}$ & jafemrSSSSSSSSSSSSSSnode & $\begin{array}{r}1232225 \\
55+00\end{array}$ & $\begin{array}{l}\mathrm{F} \\
29 \mathrm{~N} \\
12 \mathrm{~W} \\
26\end{array}$ & $\begin{array}{l}\text { A B } \\
F\end{array}$ \\
\hline $\begin{array}{c}233.54 \\
99\end{array}$ & $\begin{array}{l}\text { (Sharon CK) } \\
\text { TAPS B/G; CMP }\end{array}$ & $\begin{array}{l}\text { BB?CN? } \\
\text { GR?LS? } \\
\text { RW? }\end{array}$ & jafemrSSSSSSSSSSSSSSnode & $\begin{array}{r}1233075 \\
45+43\end{array}$ & $\begin{array}{l}\mathrm{F} \\
29 \mathrm{~N} \\
12 \mathrm{~W} \\
26\end{array}$ & $\begin{array}{ll}\mathrm{AB} & \mathrm{E} \\
\mathrm{F} & \end{array}$ \\
\hline $\begin{array}{c}234.24 \\
99\end{array}$ & $\begin{array}{l}\text { (Mary Angel CK) } \\
\text { TAPS B/G; CMP }\end{array}$ & $\begin{array}{l}\text { BB;CN; } \\
\text { GR;LS; } \\
\text { WF }\end{array}$ & jafemrCCCCCCSSSSCCCCnode & $\begin{array}{r}1236800 \\
8+47\end{array}$ & $\begin{array}{l}\mathrm{F} \\
29 \mathrm{~N} \\
12 \mathrm{~W} \\
26\end{array}$ & $\begin{array}{ll}\text { A B } & \text { E } \\
F & \end{array}$ \\
\hline $\begin{array}{c}234.31 \\
98\end{array}$ & $\begin{array}{l}\text { (South Fork, Mary Angel CK) } \\
\text { TAPS B/G; CMP }\end{array}$ & $\mathrm{CN} ; \mathrm{GR}$ & jafemrSSSSSSSSSSSSSSnode & $\begin{array}{r}1237150 \\
4+30\end{array}$ & $\begin{array}{l}\mathrm{F} \\
29 \mathrm{~N} \\
12 \mathrm{~W} \\
26\end{array}$ & $\begin{array}{ll}\text { A B } & \text { E } \\
F & \end{array}$ \\
\hline $\begin{array}{c}235.49 \\
98\end{array}$ & $\begin{array}{l}\text { (Texas Slough) } \\
\text { TAPS B/G; PARALLEL ONLY } \\
\text { TAPS DOES NOT CROSS }\end{array}$ & $\begin{array}{l}\text { CN;GR; } \\
\mathrm{KS}\end{array}$ & jafemrSSSSSSSSSSSSSSnode & $\begin{array}{l}1243275 \\
1243475 \\
1089+00 \\
1087+00\end{array}$ & $\begin{array}{l}\mathrm{F} \\
28 \mathrm{~N} \\
12 \mathrm{~W} \\
03\end{array}$ & $\begin{array}{l}\text { A B D E } \\
F\end{array}$ \\
\hline $\begin{array}{c}235.62 \\
98\end{array}$ & $\begin{array}{l}\text { (1079 Slough) } \\
\text { TAPS B/G; CMP }\end{array}$ & CN;GR & jafemrCCCCCCSSSSCCCCnode & $\begin{array}{l}1244062 \\
1079+50\end{array}$ & $\begin{array}{l}\mathrm{F} \\
28 \mathrm{~N} \\
12 \mathrm{~W} \\
03\end{array}$ & $\begin{array}{l}\mathrm{AB} \\
\mathrm{F}\end{array}$ \\
\hline $\begin{array}{c}236.07 \\
98\end{array}$ & $\begin{array}{l}\text { (Oregano CK) } \\
\text { TAPS B/G; LWC }\end{array}$ & $\begin{array}{l}\text { CN?DV; } \\
\text { GR }\end{array}$ & jafemrSSSSSSSSSSSSSSnode & $\begin{array}{l}1246450 \\
1057+00\end{array}$ & $\begin{array}{l}\mathrm{F} \\
28 \mathrm{~N} \\
12 \mathrm{~W} \\
03\end{array}$ & $\begin{array}{l}A \mathrm{~B} \\
\mathrm{~F}\end{array}$ \\
\hline $\begin{array}{c}236.17 \\
98\end{array}$ & $\begin{array}{l}\text { (Equisetum CK) } \\
\text { TAPS B/G; CMP }\end{array}$ & GR & jafemrSSSSSSSSSSSSSSnode & $\begin{array}{l}1247000 \\
1051+80\end{array}$ & $\begin{array}{l}\mathrm{F} \\
28 \mathrm{~N} \\
12 \mathrm{~W} \\
03\end{array}$ & $\begin{array}{l}\text { A B } \\
\text { F }\end{array}$ \\
\hline $\begin{array}{c}236.45 \\
98\end{array}$ & $\begin{array}{l}\text { CLARA CREEK } \\
\text { TAPS A/G; CMP }\end{array}$ & GR? & jafemrSSSSSSSSSSSSSSnode & $\begin{array}{l}1248425 \\
1248490 \\
1036+20\end{array}$ & $\begin{array}{l}\mathrm{F} \\
28 \mathrm{~N} \\
12 \mathrm{~W} \\
10\end{array}$ & I \\
\hline $\begin{array}{c}236.49 \\
98\end{array}$ & $\begin{array}{l}\text { CLARA CREEK } \\
\text { TAPS A/G; CMP }\end{array}$ & $\begin{array}{l}\text { CN?GR; } \\
\text { RW? }\end{array}$ & jafemrSSSSSSSSSSSSSSnode & $\begin{array}{l}1248648 \\
1248715 \\
1033+06\end{array}$ & $\begin{array}{l}\mathrm{F} \\
28 \mathrm{~N} \\
12 \mathrm{~W} \\
10\end{array}$ & $\begin{array}{l}\mathrm{AB} \\
\mathrm{F}\end{array}$ \\
\hline $\begin{array}{c}236.83 \\
98\end{array}$ & $\begin{array}{l}\text { South Fork, CLARA CK } \\
\text { TAPS A/G; CMP }\end{array}$ & $\begin{array}{l}\text { CN;DV? } \\
\text { GR;RW }\end{array}$ & jafemrSSSSSSSSSSSSSSnode & $\begin{array}{l}1250437 \\
1250497 \\
1015+50\end{array}$ & $\begin{array}{l}\mathrm{F} \\
28 \mathrm{~N} \\
12 \mathrm{~W} \\
10 \\
\end{array}$ & $\begin{array}{ll}A B & E \\
F\end{array}$ \\
\hline $\begin{array}{c}237.04 \\
98\end{array}$ & $\begin{array}{l}\text { (Calf CK) } \\
\text { TAPS A/G; BRIDGE }\end{array}$ & $\begin{array}{l}\text { CD?DV? } \\
\text { GR;RW? }\end{array}$ & $\begin{array}{l}\text { jafemrSSSSSSSSSSSSSSnode } \\
\end{array}$ & $\begin{array}{l}1251552 \\
1251612 \\
1004+30\end{array}$ & $\begin{array}{l}\mathrm{F} \\
28 \mathrm{~N} \\
12 \mathrm{~W} \\
10 \\
\end{array}$ & $\begin{array}{ll}\mathrm{AB} & \mathrm{E} \\
\mathrm{F} & \end{array}$ \\
\hline 237.57 & SLATE CREEK & $\mathrm{CN} ; \mathrm{DS} ;$ & $Y:$ jafemrCCCCCSSCCCCCCCnode & 1254250 & $\mathrm{~F}$ & $\mathrm{AB}$ \\
\hline
\end{tabular}




\begin{tabular}{|c|c|c|c|c|c|c|}
\hline$\& \mathrm{G}:$ & $\mathrm{Z}$ & & & $07 / 30 / 03$ & $\mathrm{P}$ & \\
\hline MP & : STREAM NAME(s) & : FISH & : A: JaFeMrApMaJuJlAuSeOcNoDe & FIELD & MER & : REFER \\
\hline $\mathrm{A} / \mathrm{S}$ & : Comments & $\begin{array}{l}\text { : SPECIES } \\
:\end{array}$ & $\begin{array}{l}\text { : D : PERIOD OF SENSITIVITY } \\
: \mathrm{A}: \\
: \mathrm{D}:\end{array}$ & $\begin{array}{l}: \text { STATION } \\
: \quad \text { G-5 }\end{array}$ & $\begin{array}{l}\text { T } \\
\text { R } \\
\text { SEC }\end{array}$ & $\begin{array}{l}:- \text { ENCE } \\
:\end{array}$ \\
\hline 98 & TAPS B/G; BLOCKPOIN'T & $\begin{array}{l}\text { DV;GR; } \\
\text { KS;RW }\end{array}$ & $\begin{array}{l}E ! \\
S\end{array}$ & $\begin{array}{r}1254450 \\
976+83\end{array}$ & $\begin{array}{l}28 \mathrm{~N} \\
12 \mathrm{~W} \\
15\end{array}$ & FG \\
\hline $\begin{array}{c}239.26 \\
98\end{array}$ & $\begin{array}{l}\text { (Horseshoe Slough) } \\
\text { TAPS A/G; CMP }\end{array}$ & GR? & jafemrSSSSSSSSSSSSSSnode & $\begin{array}{r}1263276 \\
1263344 \\
888+00\end{array}$ & $\begin{array}{l}\mathrm{F} \\
28 \mathrm{~N} \\
12 \mathrm{~W} \\
20\end{array}$ & $\mathrm{AB}$ \\
\hline $\begin{array}{c}239.76 \\
98\end{array}$ & $\begin{array}{l}\text { (Spring Slough) } \\
\text { TAPS A/G; LWC }\end{array}$ & $\mathrm{CD} / \mathrm{GR}$ & jafemrCCCCCCSSSSCCCCnode & $\begin{array}{r}1265884 \\
1265947 \\
862+10\end{array}$ & $\begin{array}{l}\mathrm{F} \\
28 \mathrm{~N} \\
12 \mathrm{~W} \\
20\end{array}$ & $\begin{array}{l}\text { A B } \\
\text { F }\end{array}$ \\
\hline $\begin{array}{c}240.13 \\
97\end{array}$ & $\begin{array}{l}\text { (East Fork, Spring Slough) } \\
\text { TAPS A/G; CMP }\end{array}$ & GR & jafemrSSSSSSSSSSSSSSnode & $\begin{array}{r}1267880 \\
1267940 \\
842+00\end{array}$ & $\begin{array}{l}\mathrm{F} \\
28 \mathrm{~N} \\
12 \mathrm{~W} \\
29\end{array}$ & $\begin{array}{l}\text { A B } \\
\text { F }\end{array}$ \\
\hline $\begin{array}{c}240.26 \\
97\end{array}$ & $\begin{array}{l}\text { (East Fork, Spring Slough) } \\
\text { TAPS A/G; LWC }\end{array}$ & GR & jafemrSSSSSSSSSSSSSSnode & $\begin{array}{r}1268559 \\
1268619 \\
835+90\end{array}$ & $\begin{array}{l}\mathrm{F} \\
28 \mathrm{~N} \\
12 \mathrm{~W} \\
29\end{array}$ & $\begin{array}{l}\text { A B } \\
\text { F }\end{array}$ \\
\hline $\begin{array}{r}240.37 \\
97\end{array}$ & $\begin{array}{l}\text { (Spring Slough) } \\
\text { TAPS A/G; CMP }\end{array}$ & CD? GR & jafemrCCCCCCSSSSCCCCnode & $\begin{array}{r}1269132 \\
1269191 \\
829+50\end{array}$ & $\begin{array}{l}\mathrm{F} \\
28 \mathrm{~N} \\
12 \mathrm{~W} \\
29\end{array}$ & $\begin{array}{l}\text { A B } \\
\text { F }\end{array}$ \\
\hline $\begin{array}{c}240.66 \\
97\end{array}$ & $\begin{array}{l}\text { (South Fork, Spring Slough) } \\
\text { TAPS A/G; CMP }\end{array}$ & $\begin{array}{l}\text { CN?GR; } \\
\text { NP }\end{array}$ & jafemrSSSSSSSSSSSSSSnode & $\begin{array}{r}1270653 \\
1270713 \\
810+00\end{array}$ & $\begin{array}{l}\mathrm{F} \\
28 \mathrm{~N} \\
12 \mathrm{~W} \\
29\end{array}$ & $\begin{array}{l}\overline{A B} \\
\text { F }\end{array}$ \\
\hline $\begin{array}{c}240.79 \\
97\end{array}$ & $\begin{array}{l}\text { (Spring Slough) } \\
\text { TAPS A/G; CMP }\end{array}$ & $\mathrm{CN} ; \mathrm{GR}$ & jafemrCCCCCCSSSSCCCCnode & $\begin{array}{r}1271357 \\
1271411 \\
804+00\end{array}$ & $\begin{array}{l}\mathrm{F} \\
28 \mathrm{~N} \\
12 \mathrm{~W} \\
29\end{array}$ & $\begin{array}{l}\text { A B } \\
\text { F }\end{array}$ \\
\hline $\begin{array}{r}242.89 \\
97\end{array}$ & $\begin{array}{l}\text { ROSIE CREEK } \\
\text { TAPS B/G; LWC }\end{array}$ & $\begin{array}{l}\text { CN?DV? } \\
\text { GR;RW? } \\
\text { KS }\end{array}$ & $\left.\begin{array}{c}Y \\
Y \\
Y\end{array}\right]$ & $\begin{array}{r}1282475 \\
694+50\end{array}$ & $\begin{array}{l}F \\
27 \mathrm{~N} \\
12 \mathrm{~W} \\
06\end{array}$ & $\begin{array}{l}\text { A B } \\
\text { F }\end{array}$ \\
\hline $\begin{array}{c}243.41 \\
97\end{array}$ & $\begin{array}{l}\text { ROSIE CREEK } \\
\text { TAPS B/G; CMP }\end{array}$ & $\begin{array}{l}\text { CN;DV; } \\
\text { GR;RW }\end{array}$ & SSSSSSCCCCCCSSSSCCCCSSSS & $\begin{array}{l}1285200 \\
666+00\end{array}$ & $\begin{array}{l}F \\
27 N \\
13 W \\
01\end{array}$ & $\begin{array}{l}\mathrm{A} \mathrm{B} \\
\mathrm{FG}\end{array}$ \\
\hline $\begin{array}{c}243.94 \\
97\end{array}$ & $\begin{array}{l}\text { (Mud CK) } \\
\text { TAPS B/G; CMP }\end{array}$ & $\mathrm{BB} ; \mathrm{GR}$ & jafemrSSSSSSSSSSSSSSnode & $\begin{array}{r}1287989 \\
1288049 \\
639+30\end{array}$ & $\begin{array}{l}F \\
27 \mathrm{~N} \\
13 \mathrm{~W} \\
12\end{array}$ & $\begin{array}{l}\mathrm{AB}, \mathrm{E} \\
\mathrm{F}\end{array}$ \\
\hline $\begin{array}{c}244.84 \\
97\end{array}$ & $\begin{array}{l}\text { (Jackson Slough) } \\
\text { TAPS A/G; CMP }\end{array}$ & $\begin{array}{l}\mathrm{CN} ; \mathrm{GR} \\
\mathrm{KS} ; \mathrm{RW}\end{array}$ & jafemrCCCCCCSSSSCCCCnode & $\begin{array}{r}1292730 \\
1292799 \\
593+00\end{array}$ & $\begin{array}{l}\mathrm{F} \\
27 \mathrm{~N} \\
13 \mathrm{~W} \\
11\end{array}$ & $\begin{array}{l}\text { A B D E } \\
\text { F }\end{array}$ \\
\hline $\begin{array}{c}245.26 \\
97\end{array}$ & $\begin{array}{l}\text { (Jackson Slough) } \\
\text { TAPS A/G; CMP }\end{array}$ & $\begin{array}{l}\mathrm{CN} ; \mathrm{GR} \\
\mathrm{KS} ; \mathrm{RW}\end{array}$ & jafemrCCCCCCSSSSCCCCnode & $\begin{array}{r}1294964 \\
1295024 \\
570+78\end{array}$ & $\begin{array}{l}F \\
27 N \\
13 W \\
14 \\
\end{array}$ & $\begin{array}{l}\text { A B D E } \\
\text { F }\end{array}$ \\
\hline
\end{tabular}


A\&G STIPULATION 2.5.3 ZONES OF RESTRICTED ACTIVITIES - KEY FISH AREAS 07/30/03 PAGE 12 of 21

\begin{tabular}{|c|c|c|c|c|c|}
\hline MP & : STREAM NAME(s) & $:$ FISH & A: JaFeMrApMaJuJlAuSeOcNoDe & FIELD & MER \\
\hline $\mathrm{A} / \mathrm{s}$ & : Comments & & A : & G-5 & $\mathrm{R}$ \\
\hline
\end{tabular}

\begin{tabular}{|c|c|c|c|c|c|c|}
\hline $\begin{array}{c}245.53 \\
96\end{array}$ & $\begin{array}{l}\text { (Jackson Slough) } \\
\text { TAPS A/G; BLOCKPOINT }\end{array}$ & $\begin{array}{l}\text { CN;GR; } \\
\text { KS?RW }\end{array}$ & jafemrCCCCCCSSSSCCCCnode & $\begin{array}{r}1296343 \\
1296403 \\
555+85\end{array}$ & $\begin{array}{l}\mathrm{F} \\
27 \mathrm{~N} \\
13 \mathrm{~W} \\
14 \\
\end{array}$ & $\begin{array}{l}\mathrm{AB} D \mathrm{E} \\
\mathrm{F}\end{array}$ \\
\hline $\begin{array}{c}245.60 \\
96\end{array}$ & $\begin{array}{l}\text { (Jackson Slough) } \\
\text { Fish in low reach of slough } \\
\text { TAPS A/G; BLOCKPOINT }\end{array}$ & $\begin{array}{l}\text { CN;GR; } \\
\mathrm{KS}\end{array}$ & jafemrCCCCCCSSSSCCCCnode & 1296363 & $\begin{array}{l}\mathrm{F} \\
27 \mathrm{~N} \\
13 \mathrm{~W} \\
14\end{array}$ & $\mathbf{J}$ \\
\hline $\begin{array}{c}246.26 \\
96\end{array}$ & $\begin{array}{l}\text { (Trent's Trickle) } \\
\text { TAPS A/G; CMP }\end{array}$ & $\begin{array}{l}\text { CN;GR; } \\
\text { NP? }\end{array}$ & SSSSSSCCCCCCSSSSCCCCSSSS & $\begin{array}{r}1300218 \\
1300273 \\
518+39\end{array}$ & $\begin{array}{l}\mathrm{F} \\
27 \mathrm{~N} \\
13 \mathrm{~W} \\
23 \\
\end{array}$ & $\begin{array}{l}\text { A B } \\
\text { F }\end{array}$ \\
\hline $\begin{array}{c}248.19 \\
96\end{array}$ & $\begin{array}{l}\text { (North Fork, Windy Arm } \\
\text { CK) } \\
\text { TAPS A/G; BLOCKPOINT }\end{array}$ & $\begin{array}{l}\text { CN;GR; } \\
\text { NP? }\end{array}$ & jafemrSSSSSSSSSSSSSSnode & $\begin{array}{r}1310328 \\
1310508 \\
417+25\end{array}$ & $\begin{array}{l}\mathrm{F} \\
27 \mathrm{~N} \\
13 \mathrm{~W} \\
26 \\
\end{array}$ & $\begin{array}{l}\text { A B } \\
\text { F }\end{array}$ \\
\hline $\begin{array}{c}250.50 \\
96\end{array}$ & $\begin{array}{l}\text { (Chapman CK) } \\
\text { TAPS A/C; LWC }\end{array}$ & $\begin{array}{l}\text { CD?GR; } \\
\text { NP? }\end{array}$ & jafemrSSSSSSSSSSSSSSnode & $\begin{array}{r}1322631 \\
1322691 \\
295+17\end{array}$ & $\begin{array}{l}\mathrm{F} \\
26 \mathrm{~N} \\
13 \mathrm{~W} \\
11 \\
\end{array}$ & $\begin{array}{l}\mathrm{AB} \\
\mathrm{F}\end{array}$ \\
\hline $\begin{array}{c}256.26 \\
256.36 \\
94\end{array}$ & $\begin{array}{l}\text { South Fork, KOYUKUK } \\
\text { RIVER } \\
\text { TAPS A/G; BLOCKPOINT }\end{array}$ & $\begin{array}{l}\text { BB?BW? } \\
\text { CN;DS; } \\
\text { GR;HW? } \\
\text { KS;LS; } \\
\text { NP?RW; } \\
\text { SK? }\end{array}$ & $\begin{array}{l}\mathrm{CCCCCCCCCCCCCCCCCCCCCCCC} \\
\mathrm{Y} \\
\mathrm{E}\end{array}$ & $\begin{array}{l}1353037 \\
1353602 \\
1075+15 \\
1069+68\end{array}$ & $\begin{array}{l}\mathrm{F} \\
25 \mathrm{~N} \\
12 \mathrm{~W} \\
06\end{array}$ & $\begin{array}{l}\mathrm{AB} \\
\mathrm{F}\end{array}$ \\
\hline $\begin{array}{r}258.39 \\
94\end{array}$ & $\begin{array}{l}\text { (Aba-Dabba CK) } \\
\text { TAPS A/G; CMP }\end{array}$ & $\mathrm{CN} ; \mathrm{GR}$ & SSSSSSSSSSSSSSSSSSSSSSSS & $\begin{array}{r}1364290 \\
1364356 \\
963+13\end{array}$ & $\begin{array}{l}\mathrm{F} \\
25 \mathrm{~N} \\
13 \mathrm{~W} \\
13 \\
\end{array}$ & $\begin{array}{l}\text { A B } \\
\text { F }\end{array}$ \\
\hline $\begin{array}{l}268.12 \\
92\end{array}$ & $\begin{array}{l}\text { JIM RIVER \#3 } \\
\text { TAPS B/G; BLOCKPOINT }\end{array}$ & $\begin{array}{l}\text { BB;CN; } \\
\text { DS;GR; } \\
\text { HW;KS; } \\
\text { LS;NP; } \\
\text { RW } \\
\end{array}$ & $\begin{array}{l}\text { CCCCCCCCCCCCCCCCCCCCCCCC } \\
\mathrm{E} \\
\mathrm{S}\end{array}$ & $\begin{array}{r}1415550 \\
1415800 \\
453+50\end{array}$ & $\begin{array}{l}\mathrm{F} \\
24 \mathrm{~N} \\
14 \mathrm{~W} \\
23,26\end{array}$ & $\begin{array}{l}\text { A B } \\
\text { F G }\end{array}$ \\
\hline $\begin{array}{c}268.44 \\
92\end{array}$ & $\begin{array}{l}\text { (Beaver Spring) } \\
\text { TAPS A/G; CMP }\end{array}$ & $\begin{array}{l}\text { CN?GR? } \\
\text { KS?RW? }\end{array}$ & CCCCCCCCCCCCSSSSCCCCCCCC & $\begin{array}{r}1417312 \\
1417426 \\
435+84\end{array}$ & $\begin{array}{l}\mathrm{F} \\
24 \mathrm{~N} \\
14 \mathrm{~W} \\
26 \\
\end{array}$ & \\
\hline $\begin{array}{c}269.00 \\
92\end{array}$ & $\begin{array}{l}\text { (Dee CK) } \\
\text { TAPS A/G; CMP }\end{array}$ & $\begin{array}{l}\mathrm{CN} ; \mathrm{DV} ? \\
\mathrm{GR} ; \mathrm{RW}\end{array}$ & SSSSSSSSSSSSSSSSSSSSSSSS & $\begin{array}{r}1420293 \\
1420353 \\
406+86\end{array}$ & $\begin{array}{l}\mathrm{F} \\
24 \mathrm{~N} \\
14 \mathrm{~W} \\
26 \\
\end{array}$ & $\begin{array}{l}\text { A. B } \\
\text { F }\end{array}$ \\
\hline $\begin{array}{c}270.45 \\
92\end{array}$ & $\begin{array}{l}\text { DOUGLAS CREEK } \\
\text { TAPS A/G; LWC }\end{array}$ & $\begin{array}{l}\mathrm{CN} ; \mathrm{GR} \text {; } \\
\mathrm{RW} \text { ? }\end{array}$ & jafemrSSSSSSSSSSSSSSnode & $\begin{array}{r}1427745 \\
1428168 \\
333+75\end{array}$ & $\begin{array}{l}\mathrm{F} \\
24 \mathrm{~N} \\
14 \mathrm{~W} \\
34 \\
\end{array}$ & $\begin{array}{l}\text { A. B } \\
\text { F }\end{array}$ \\
\hline $\begin{array}{c}271.62 \\
92\end{array}$ & $\begin{array}{l}\text { Side Channel JIM RIVER \#2 } \\
\text { TAPS B/G; BLOCKPOINT }\end{array}$ & $\begin{array}{l}\text { BB;CN;DS } \\
\text { GR;HW; } \\
\text { KS:LS; } \\
\text { NP;RW }\end{array}$ & $\begin{array}{l:l}Y & \mathrm{CCCCCCCCCCCCCCCCCCCCCCCC} \\
\mathrm{E}^{\prime} & \end{array}$ & $\begin{array}{r}1434078 \\
1434178 \\
272+51\end{array}$ & $\begin{array}{l}\mathrm{F} \\
23 \mathrm{~N} \\
14 \mathrm{~W} \\
03 \\
\end{array}$ & $\begin{array}{l}\text { A B } \\
\text { F }\end{array}$ \\
\hline $\begin{array}{c}271.92 \\
92\end{array}$ & $\begin{array}{l}\text { Side Channel JIM RIVER \#1 } \\
\text { TAPS B/G; BLOCKPOINT }\end{array}$ & $\begin{array}{l}\mathrm{BB} ; \mathrm{CN} ; \mathrm{DS} ; \\
\mathrm{GR} ; \mathrm{HW} \\
\mathrm{KS} ; \mathrm{LS} ; \\
\mathrm{NP} ; \mathrm{RW}\end{array}$ & $\begin{array}{l:l}Y & C C C C C C C C C C C C C C C C C C C C C C C C \\
E & \end{array}$ & $\begin{array}{r}1435700 \\
1435800 \\
257+00\end{array}$ & $\begin{array}{l}\mathrm{F} \\
24 \mathrm{~N} \\
14 \mathrm{~W} \\
09 \\
\end{array}$ & $\begin{array}{l}\text { A B } \\
\text { F }\end{array}$ \\
\hline
\end{tabular}


A\&G STIPULATION 2.5.3 ZONES OF RESTRICTED ACTIVITIES - KEY FISH AREAS 07/30/03 PAGE 13 of 21

\begin{tabular}{|c|c|c|c|c|c|c|}
\hline MP & : STREAM NAME(s) & $\begin{array}{l}\text { : FISH } \\
\text { SPECIES }\end{array}$ & $\begin{array}{l}\text { A: JaFeMrApMaJuJlAuSeOcNoDe } \\
\text { D : PERIOD OF SENSITIVITY }\end{array}$ & $\begin{array}{l}\text { : FIELD } \\
\text { STAT1ON }\end{array}$ & $\begin{array}{l}: \text { MER } \\
: T\end{array}$ & $\begin{array}{l}\text { : REFER } \\
:- \text { ENCE }\end{array}$ \\
\hline $\mathrm{A} /$ & $\begin{array}{l}\text { : Comments } \\
:\end{array}$ & $:$ & $\begin{array}{l}: \mathrm{A}: \\
: \mathrm{D}:\end{array}$ & $:$ G-5 & $\begin{array}{l}: \mathrm{R} \\
: \mathrm{SEC}\end{array}$ & \\
\hline
\end{tabular}

\begin{tabular}{|c|c|c|c|c|c|c|c|}
\hline 272.21 & $\begin{array}{l}\text { (Little Piddler) } \\
\text { TAPS A/G; CMP }\end{array}$ & GR & jafemrSSSSSSSSSSSSSSnode & $\begin{array}{r}1437254 \\
1437307 \\
240+85\end{array}$ & $\begin{array}{l}\mathrm{F} \\
23 \mathrm{~N} \\
14 \mathrm{~W} \\
09\end{array}$ & $\begin{array}{l}\text { A B } \\
\text { F }\end{array}$ & \\
\hline $\begin{array}{c}277.14 \\
91\end{array}$ & $\begin{array}{l}\text { PROSPECT CREEK } \\
\text { TAPS A/G; BLOCKPOINT }\end{array}$ & $\begin{array}{l}\text { CN;GR; } \\
\text { KS;LS; } \\
\text { NP;RW }\end{array}$ & $\begin{array}{l:l}\mathrm{CCCCCCCCCCCCCCCCCCCCCCCC} \\
\mathrm{E}\end{array}$ & $\begin{array}{l}1463150 \\
1463408 \\
1590+00\end{array}$ & $\begin{array}{l}\mathrm{F} \\
23 \mathrm{~N} \\
14 \mathrm{~W} \\
31 \\
\end{array}$ & $\begin{array}{l}\text { A B } \\
\text { F G }\end{array}$ & \\
\hline $\begin{array}{r}281.88 \\
90\end{array}$ & $\begin{array}{l}\text { (Little Nasty CK) } \\
\text { TAPS A/G; BLOCKPOINT }\end{array}$ & $\begin{array}{l}\text { CN;GR; } \\
\text { RW }\end{array}$ & jafemrSSSSSSSSSSSSSSnode & $\begin{array}{l}1488219 \\
1488452 \\
1339+00\end{array}$ & $\begin{array}{l}\mathrm{F} \\
22 \mathrm{~N} \\
15 \mathrm{~W} \\
19\end{array}$ & $\begin{array}{l}A B \\
F\end{array}$ & \\
\hline $\begin{array}{c}282.14 \\
90\end{array}$ & $\begin{array}{l}\text { (South Fork, Little Nasty CK) } \\
\text { TAPS A/G; CMP }\end{array}$ & CD?GR & jafemrSSSSSSSSSSSSSSnode & $\begin{array}{l}1489653 \\
1489705 \\
1327+40\end{array}$ & $\begin{array}{l}\mathrm{F} \\
22 \mathrm{~N} \\
15 \mathrm{~W} \\
19\end{array}$ & $\begin{array}{l}\text { A B } \\
\text { F }\end{array}$ & \\
\hline $\begin{array}{c}284.40 \\
89\end{array}$ & $\begin{array}{l}\text { North Fork BONANZA } \\
\text { CREEK } \\
\text { TAPS B/G; BLOCKPOINT }\end{array}$ & $\begin{array}{l}\text { BB;CN; } \\
\text { GR;HW? } \\
\text { LS;:LW; } \\
\text { NP;RW }\end{array}$ & jafemrCCCCCCSSSSCCCCnode & $\begin{array}{l}1501503 \\
1501783 \\
1208+32\end{array}$ & $\begin{array}{l}\mathrm{F} \\
22 \mathrm{~N} \\
14 \mathrm{~W} \\
32 \\
\end{array}$ & $\begin{array}{l}\text { A B } \\
\text { F G }\end{array}$ & \\
\hline $\begin{array}{c}285.53 \\
89\end{array}$ & $\begin{array}{l}\text { (Oxbow Lake System) } \\
\text { TAPS B/G; CMP }\end{array}$ & GR? & jafemrCCCCCCSSauseocnode & $\begin{array}{l}1507600 \\
1148+00\end{array}$ & $\begin{array}{l}\mathrm{F} \\
21 \mathrm{~N} \\
14 \mathrm{~W} \\
07\end{array}$ & $\begin{array}{l}\text { A B } \\
\text { F }\end{array}$ & \\
\hline $\begin{array}{c}286.01 \\
89\end{array}$ & $\begin{array}{l}\text { South Fork BONANZA } \\
\text { CREEK } \\
\text { TAPS B/G; BLOCKPOINT }\end{array}$ & $\begin{array}{l}\text { BB;CN; } \\
\text { GR;HW? } \\
\text { LS;NP; } \\
\text { RW }\end{array}$ & SSSSSSCCCCCCSSSCCCCCCCSS & $\begin{array}{l}1510050 \\
1510200 \\
1123+60\end{array}$ & $\begin{array}{l}\mathrm{F} \\
21 \mathrm{~N} \\
14 \mathrm{~W} \\
07\end{array}$ & $\begin{array}{l}\text { A B } \\
\text { F G }\end{array}$ & \\
\hline $\begin{array}{c}289.63 \\
88\end{array}$ & $\begin{array}{l}\text { (Pung's Crossing CK) } \\
\text { TAPS A/G; BRIDGE }\end{array}$ & $\begin{array}{l}\text { CD?GR; } \\
\text { RW? }\end{array}$ & jafemrCCCCCCSSSSCCCCnode & $\begin{array}{r}1529195 \\
1529325 \\
932+60 \\
932+10\end{array}$ & $\begin{array}{l}\mathrm{F} \\
21 \mathrm{~N} \\
14 \mathrm{~W} \\
31\end{array}$ & $\begin{array}{l}\text { A B } \\
\text { F }\end{array}$ & $\mathrm{E}$ \\
\hline $\begin{array}{c}293.22 \\
88\end{array}$ & $\begin{array}{l}\text { (Alder Mountain CK) } \\
\text { TAPS B/G; LWC }\end{array}$ & $\begin{array}{l}\text { CN;GR? } \\
\text { RW? }\end{array}$ & jafemrSSSSSSSSSSSSSSnode & $\begin{array}{r}1548200 \\
742+50\end{array}$ & $\begin{array}{l}\mathrm{F} \\
20 \mathrm{~N} \\
15 \mathrm{~W} \\
10 \\
\end{array}$ & $\begin{array}{l}\text { A B } \\
\text { F }\end{array}$ & $\mathrm{E}$ \\
\hline $\begin{array}{c}294.91 \\
88\end{array}$ & $\begin{array}{l}\text { FISH CREEK } \\
\text { TAPS A/G; BRIDGE }\end{array}$ & $\begin{array}{l}\text { BW?CN; } \\
\text { DS?GR; } \\
\text { LS;NP? } \\
\text { RW;SK }\end{array}$ & jafemrCCCCCCSSSSCCCCnode & $\begin{array}{r}1557094 \\
1557163 \\
653+50\end{array}$ & $\begin{array}{l}\mathrm{F} \\
20 \mathrm{~N} \\
15 \mathrm{~W} \\
22 \\
\end{array}$ & $\begin{array}{l}\mathrm{A} \mathrm{B} \\
\mathrm{F}\end{array}$ & \\
\hline $\begin{array}{c}296.34 \\
87\end{array}$ & $\begin{array}{l}\text { Middle Fork, FISH CREEK } \\
\text { TAPS A/G; CMP }\end{array}$ & $\begin{array}{l}\text { CN;GR; } \\
\text { RW }\end{array}$ & jafemrSSSSSSSSSSSSSSnode & $\begin{array}{r}1564650 \\
578+00\end{array}$ & $\begin{array}{l}\mathrm{F} \\
20 \mathrm{~N} \\
15 \mathrm{~W} \\
26 \\
\end{array}$ & $\begin{array}{l}\text { A B } \\
\text { F }\end{array}$ & \\
\hline $\begin{array}{c}297.42 \\
87\end{array}$ & $\begin{array}{l}\text { South Fork FISH CREEK } \\
\text { TAPS A/G; BRIDGE }\end{array}$ & $\begin{array}{l}\text { CN;GR; } \\
\text { RW? }\end{array}$ & SSSSSSCCCCCCSSSSCCCCCCSS & $\begin{array}{r}1570361 \\
1570423 \\
520+60\end{array}$ & $\begin{array}{l}\mathrm{F} \\
20 \mathrm{~N} \\
15 \mathrm{~W} \\
35 \\
\end{array}$ & $\begin{array}{l}\text { A B } \\
\text { F }\end{array}$ & \\
\hline $\begin{array}{c}302.92 \\
86 \\
\end{array}$ & $\begin{array}{l}\text { KANUTI RIVER } \\
\text { TAPS B/G; BLOCKPOINT }\end{array}$ & $\begin{array}{l}\text { BB;BC? } \\
\text { BW?CN; } \\
\text { CS?DS? } \\
\text { GR;HW? } \\
\text { IN?LS? } \\
\text { NP;RW }\end{array}$ & jafemrCCCCCCSSSSCCCCnode & $\begin{array}{r}1599300 \\
1599500 \\
231+00\end{array}$ & $\begin{array}{l}\mathrm{F} \\
19 \mathrm{~N} \\
14 \mathrm{~W} \\
30\end{array}$ & $\begin{array}{l}\mathrm{AB} \\
\mathrm{F}\end{array}$ & \\
\hline
\end{tabular}


A\&G STIPULATION 2.5.3 ZONES OF RESTRICTED ACTIVITIES - KEY FISH AREAS

\begin{tabular}{|c|c|c|c|c|c|c|}
\hline MP & $\begin{array}{l}\text { : STREAM NAME(s) } \\
:\end{array}$ & $\begin{array}{l}\text { : FISH } \\
: \text { SPECIES }\end{array}$ & $\begin{array}{l}\text { : A: JaFeMrApMaJuJlAuSeOcNoDe } \\
: \text { D : PERIOD OF SENSITIVITY }\end{array}$ & $\begin{array}{l}\text { : FIELD } \\
\text { : STATION }\end{array}$ & $\begin{array}{l}\text { : MER } \\
: \mathrm{T}\end{array}$ & $\begin{array}{l}\text { : REFER } \\
:- \text { ENCE }\end{array}$ \\
\hline A/S & : Comments & : & $: \mathrm{A}:$ & G-5 & $: \mathbf{R}$ & : \\
\hline & : & : & : D : & : & : SEC & : \\
\hline
\end{tabular}

\begin{tabular}{|c|c|c|c|c|c|c|}
\hline $\begin{array}{c}306.22 \\
86\end{array}$ & $\begin{array}{l}\text { (Caribou Mountain CK) } \\
\text { TAPS B.G; BLOCKPOINT }\end{array}$ & GR & jafemrSSSSSSSSSSSSSSnode & $\begin{array}{r}1616819 \\
1616887 \\
56+03\end{array}$ & $\begin{array}{l}\mathrm{F} \\
18 \mathrm{~N} \\
14 \mathrm{~W} \\
09\end{array}$ & $\begin{array}{l}\mathrm{AB} B \\
\mathrm{~F}\end{array}$ \\
\hline $\begin{array}{c}308.56 \\
85\end{array}$ & $\begin{array}{l}\text { (Olson's Lake CK) } \\
\text { TAPS A/G; BLOCKPOINT }\end{array}$ & GR & jafemrSSSSSSSSSSSSSSnode & $\begin{array}{l}1629150 \\
1629200 \\
1149+39\end{array}$ & $\begin{array}{l}\mathrm{F} \\
18 \mathrm{~N} \\
14 \mathrm{~W} \\
14 \\
\end{array}$ & $\begin{array}{l}\text { A B } \\
\text { F }\end{array}$ \\
\hline $\begin{array}{c}312.13 \\
85\end{array}$ & $\begin{array}{l}\text { (Finger Mountain CK) } \\
\text { TAPS A.G; LWC }\end{array}$ & GR & jafemrSSSSSSSSSSSSSSnode & $\begin{array}{r}1648020 \\
1648050 \\
961+80\end{array}$ & $\begin{array}{l}\mathrm{F} \\
18 \mathrm{~N} \\
14 \mathrm{~W} \\
36\end{array}$ & $\begin{array}{l}\mathrm{AB} \\
\mathrm{F}\end{array}$ \\
\hline $\begin{array}{c}315.24 \\
84\end{array}$ & $\begin{array}{l}\text { Middle Branch, West Fork } \\
\text { DALL RIVER } \\
\text { TAPS A/G; LWC }\end{array}$ & $\begin{array}{l}\text { CD?GR; } \\
\text { IN?WF? }\end{array}$ & jajafemrSSSSSSSSSSSSSSnode & $\begin{array}{r}1664436 \\
1664496 \\
798+00\end{array}$ & $\begin{array}{l}\mathrm{F} \\
17 \mathrm{~N} \\
13 \mathrm{~W} \\
17\end{array}$ & $\begin{array}{l}A B \\
F\end{array}$ \\
\hline $\begin{array}{c}317.59 \\
84\end{array}$ & $\begin{array}{l}\text { South Branch, West Fork } \\
\text { DALL RIVER } \\
\text { TAPS A/G; BLOCKPOINT }\end{array}$ & $\begin{array}{l}\text { CD?GR } \\
\text { IN?WF? }\end{array}$ & jafemrSSSSSSSSSSSSSSnode & $\begin{array}{r}1676845 \\
1676914 \\
673+00\end{array}$ & $\begin{array}{l}\mathrm{F} \\
17 \mathrm{~N} \\
13 \mathrm{~W} \\
28 \\
\end{array}$ & $\begin{array}{l}\text { A B } \\
\text { F }\end{array}$ \\
\hline $\begin{array}{c}325.25 \\
82\end{array}$ & $\begin{array}{l}\text { (Fed CK) } \\
\text { TAPS A/G; LWC }\end{array}$ & CD?GR & jafemrSSSSSSSSSSSSSSnode & $\begin{array}{r}1717311 \\
1717379 \\
270+10\end{array}$ & $\begin{array}{l}\mathrm{F} \\
16 \mathrm{~N} \\
13 \mathrm{~W} \\
25 \\
\end{array}$ & $\begin{array}{l}\text { A B } \\
F\end{array}$ \\
\hline $\begin{array}{c}329.29 \\
82\end{array}$ & $\begin{array}{l}\text { North Fork, RAY RIVER } \\
\text { No Name CK) } \\
\text { TAPS A/G; BLOCKPOINT }\end{array}$ & $\begin{array}{l}\text { BB;CN; } \\
\text { GR;IN? } \\
\text { LC?LS } \\
\text { NP;RW }\end{array}$ & jafemrCCCCCCSSSSCCCCCCde & $\begin{array}{r}1738635 \\
1738705 \\
58+00\end{array}$ & $\begin{array}{l}\mathrm{F} \\
15 \mathrm{~N} \\
12 \mathrm{~W} \\
17 \\
\end{array}$ & $\begin{array}{l}\text { A B } \\
F\end{array}$ \\
\hline $\begin{array}{c}336.18 \\
81\end{array}$ & $\begin{array}{l}\text { (Fort Hamlin Hills CK) } \\
\text { TAPS A/G; BLOCKPOINT }\end{array}$ & $\begin{array}{l}\text { CD?GR; } \\
\text { RW? }\end{array}$ & jafemrSSSSSSSSSSSSSSnode & $\begin{array}{r}1775006 \\
1775045 \\
971+30\end{array}$ & $\begin{array}{l}\mathrm{F} \\
14 \mathrm{~N} \\
12 \mathrm{~W} \\
17 \\
\end{array}$ & $\begin{array}{l}\mathrm{AB} \\
\mathrm{F}\end{array}$ \\
\hline $\begin{array}{c}344.99 \\
79\end{array}$ & $\begin{array}{l}\text { (Phelps CK) } \\
\text { TAPS A/G; LWC }\end{array}$ & GR & jafemrSSSSSSSSSSSSSSnode & $\begin{array}{r}1821536 \\
1821595 \\
508+38\end{array}$ & $\begin{array}{l}\mathrm{F} \\
13 \mathrm{~N} \\
11 \mathrm{~W} \\
17 \\
\end{array}$ & $\begin{array}{l}\text { A B E } \\
\text { F }\end{array}$ \\
\hline $\begin{array}{c}350.50 \\
78\end{array}$ & $\begin{array}{l}\text { (Wood Chopper CK) } \\
\text { TAPS A/G; BLOCKPOINT }\end{array}$ & $\begin{array}{l}\text { CD?GR; } \\
\text { NP?WF? }\end{array}$ & jafemrSSSSSSSSSSSSSSnode & $\begin{array}{r}1850595 \\
1850656 \\
215+81\end{array}$ & $\begin{array}{l}\mathrm{F} \\
13 \mathrm{~N} \\
11 \mathrm{~W} \\
36\end{array}$ & $\begin{array}{lll}\text { A B } \quad \text { E } \\
\text { F } \\
\end{array}$ \\
\hline $\begin{array}{c}351.58 \\
78\end{array}$ & $\begin{array}{l}\text { (Burbot } \mathrm{CK} \text { ) } \\
\text { TAPS A/G; CMP }\end{array}$ & $\mathrm{BB}$ & jafemrapSSSSSSSSSSSSSSnode & $\begin{array}{r}1856332 \\
1856396 \\
158+21\end{array}$ & $\begin{array}{l}\mathrm{F} \\
12 \mathrm{~N} \\
11 \mathrm{~W} \\
01 \\
\end{array}$ & $\begin{array}{ll}A B & \text { E } \\
F & \end{array}$ \\
\hline $\begin{array}{c}353.07 \\
353.50 \\
78\end{array}$ & $\begin{array}{l}\text { YUKON RIVER } \\
\text { TAPS A/G; BRIDGE }\end{array}$ & $\begin{array}{l}\text { AL;BB; } \\
\text { BC;BL? } \\
\text { BW;CA? } \\
\text { CN;CS; } \\
\text { DS;GR; } \\
\text { HO?HW; } \\
\text { IN;KS; } \\
\text { LC;LS; } \\
\text { LW;NP; } \\
\text { PS;RS; } \\
\text { RW;SS }\end{array}$ & $\begin{array}{l:l} & \mathrm{CCCCCCCCCCCCCCCCCCCCCCCC} \\
\mathrm{E} & \end{array}$ & $\begin{array}{r}1864200 \\
1866500 \\
78+97 \\
60+00\end{array}$ & $\begin{array}{l}\text { F12N } \\
10 W \\
07\end{array}$ & $\begin{array}{ll}\mathrm{AB} & \mathrm{E} \\
\mathrm{F}\end{array}$ \\
\hline
\end{tabular}


A\&G STIPULATION 2.5.3 ZONES OF RESTRICTED ACTIVITIES - KEY FISH AREAS 07/30/03 PAGE 15 of 21

\begin{tabular}{|c|c|c|c|c|c|c|}
\hline MP & : STREAM NAME(s) & :FISH & A: JaFeMrApMaJuJlAuSeOcNoDe & :FIELD & :MER & $\begin{array}{l}\text { EFER } \\
\text { ENCE }\end{array}$ \\
\hline $\mathrm{A} / \mathrm{S}$ & Comments & $\begin{array}{l}: \text { SPECIES } \\
:\end{array}$ & $\begin{array}{l}: \text { D : PERIOD OF SENSITIV } \\
: \text { A : }\end{array}$ & $\begin{array}{l}: \text { STATION } \\
: \quad \text { G-5 }\end{array}$ & & \\
\hline
\end{tabular}

\begin{tabular}{|c|c|c|c|c|c|c|}
\hline $\begin{array}{c}362.72 \\
76\end{array}$ & $\begin{array}{l}\text { ISOM CREEK } \\
\text { TAPS A/G; } \\
\text { SUMMER BLOCKPOINT }\end{array}$ & $\overline{\mathrm{GR}}$ & jafemrapSSSSSSSSSSSSnode & $\begin{array}{l}1915150 \\
1651+65\end{array}$ & $\begin{array}{l}\mathrm{F} \\
11 \mathrm{~N} \\
09 \mathrm{~W} \\
07\end{array}$ & $\begin{array}{ll}\mathrm{A} B & \mathrm{E} \\
\mathrm{F} & \end{array}$ \\
\hline $\begin{array}{c}362.76 \\
76\end{array}$ & $\begin{array}{l}\text { ISOM CREEK } \\
\text { TAPS AVG; } \\
\text { SUMMER BLOCKPOINT }\end{array}$ & GR & jafemrapSSSSSSSSSSSSnode & $\begin{array}{l}1915400 \\
1642+39\end{array}$ & $\begin{array}{l}\mathrm{F} \\
11 \mathrm{~N} \\
09 \mathrm{~W} \\
07\end{array}$ & $\begin{array}{ll}\text { A B } & \text { E } \\
\text { F } & \end{array}$ \\
\hline $\begin{array}{c}370.53 \\
75\end{array}$ & $\begin{array}{l}(\text { Hot Cat CK) } \\
\text { TAPS A/G; CMP }\end{array}$ & GR & jafemrapSSSSSSSSSSSSnode & $\begin{array}{l}1956346 \\
1956416 \\
1242+46\end{array}$ & $\begin{array}{l}\mathrm{F} \\
11 \mathrm{~N} \\
08 \mathrm{~W} \\
31\end{array}$ & $\begin{array}{ll}\text { A B } & \text { E } \\
\text { F } & \end{array}$ \\
\hline $\begin{array}{c}378.39 \\
73\end{array}$ & $\begin{array}{l}\text { FISH CREEK } \\
\text { TAPS A/G; BRIDGE }\end{array}$ & $\begin{array}{l}\text { CN?GR; } \\
\text { NP;WF }\end{array}$ & jafemrapSSSSSSSSSSSSnode & $\begin{array}{c}1997824 \\
1997932 \\
829+56\end{array}$ & $\begin{array}{l}\mathrm{F} \\
10 \mathrm{~N} \\
07 \mathrm{~W} \\
20\end{array}$ & $\begin{array}{ll}\mathrm{AB} & \mathrm{E} \\
\mathrm{F} & \end{array}$ \\
\hline $\begin{array}{c}378.57 \\
73\end{array}$ & $\begin{array}{l}\text { HESS CREEK } \\
\text { TAPS A/G; BLOCKPOINT }\end{array}$ & $\begin{array}{l}\text { AL?BC; } \\
\text { BW;CN; } \\
\text { CS;DS? } \\
\text { GR;HW; } \\
\text { IN;LS; } \\
\text { NP;RW }\end{array}$ & jafemrCCCCCCCCCCCCCCnode & $\begin{array}{r}1998734 \\
1998928 \\
820+49 \\
819+31\end{array}$ & $\begin{array}{l}\mathrm{F} \\
10 \mathrm{~N} \\
07 \mathrm{~W} \\
20\end{array}$ & $\begin{array}{ll}\text { A B } & \text { E } \\
\text { F } & \end{array}$ \\
\hline $\begin{array}{c}382.50 \\
73\end{array}$ & $\begin{array}{l}\text { ERICKSON CREEK \# } 2 \\
\text { TAPS A/G; BRIDGE }\end{array}$ & $\begin{array}{l}\text { CN?GR; } \\
\text { LS }\end{array}$ & jafemrapSSSSSSSSSSSSnode & $\begin{array}{r}2019562 \\
2019642 \\
611+95\end{array}$ & $\begin{array}{l}\mathrm{F} \\
09 \mathrm{~N} \\
07 \mathrm{~W} \\
03\end{array}$ & $\begin{array}{ll}\text { A B } & \text { E } \\
\text { F } & \end{array}$ \\
\hline $\begin{array}{c}384.37 \\
72\end{array}$ & $\begin{array}{l}\text { ERICKSON CREEK \# } 1 \\
\text { TAPS A/G; BRIDGE }\end{array}$ & $\begin{array}{l}\text { CN?GR; } \\
\text { LS }\end{array}$ & jafemrapSSSSSSSSSSSSnode & $\begin{array}{r}2029448 \\
2029517 \\
513+62\end{array}$ & $\begin{array}{l}\mathrm{F} \\
09 \mathrm{~N} \\
07 \mathrm{~W} \\
14\end{array}$ & $\begin{array}{ll}A B & E \\
F\end{array}$ \\
\hline $\begin{array}{c}392.05 \\
71\end{array}$ & $\begin{array}{l}\text { LOST CREEK } \\
\text { TAPS A/G; BRIDGE }\end{array}$ & $\begin{array}{l}\mathrm{CN} ; \mathrm{GR}_{i} \\
\mathrm{WF}\end{array}$ & jafemrapSSSSSSSSSSSSnode & $\begin{array}{r}2069975 \\
2070055 \\
104+33\end{array}$ & $\begin{array}{l}\mathrm{F} \\
08 \mathrm{~N} \\
06 \mathrm{~W} \\
16\end{array}$ & $\begin{array}{ll}A \text { B } & \text { E } \\
F & \end{array}$ \\
\hline $\begin{array}{c}398.56 \\
70\end{array}$ & $\begin{array}{l}\text { TOLOVANA RIVER } \\
\text { TAPS AJG; BRIDGE }\end{array}$ & $\begin{array}{l}\text { AB?BB; } \\
\text { CI?CN? } \\
\text { CS?DS; } \\
\text { GR;HW; } \\
\text { IN;KS; } \\
\text { LW?NP; } \\
\text { SS? }\end{array}$ & jafemrapSSSSSSSSSSSSSSSS & $\begin{array}{l}2104372 \\
2104470 \\
2958+13 \\
2957+13\end{array}$ & $\begin{array}{l}\mathrm{F} \\
07 \mathrm{~N} \\
05 \mathrm{~W} \\
05\end{array}$ & $\begin{array}{ll}A \text { B } & \text { E } \\
F & \end{array}$ \\
\hline $\begin{array}{c}405.51 \\
68\end{array}$ & $\begin{array}{l}\text { WILBER CREEK } \\
\text { TAPS B/G; CMP }\end{array}$ & GR & JafemrapSSSSSSSSSSSSnode & $\begin{array}{l}2141100 \\
2609+50\end{array}$ & $\begin{array}{l}\mathrm{F} \\
07 \mathrm{~N} \\
04 \mathrm{~W} \\
30\end{array}$ & $\begin{array}{ll}A B & E \\
F & \end{array}$ \\
\hline $\begin{array}{c}408.36 \\
68\end{array}$ & $\begin{array}{l}\text { SLATE CREEK } \\
\text { TAPS A/G; CMP }\end{array}$ & GR & jafemrapSSSSSSSSSSSSnode & $\begin{array}{l}2156110 \\
2156181 \\
2459+42\end{array}$ & $\begin{array}{l}\mathrm{F} \\
06 \mathrm{~N} \\
04 \mathrm{~W} \\
5\end{array}$ & $\begin{array}{ll}A \text { B } & \text { E } \\
F & \end{array}$ \\
\hline 412.50 & $\begin{array}{l}\text { TATALINA RIVER } \\
\text { TAPS A/G; BLOCKPOINT }\end{array}$ & $\begin{array}{l}\mathrm{BB} ? \mathrm{Cl} \\
\text { CN?GR; } \\
\text { IN?NP; } \\
\text { SK?WF }\end{array}$ & jafemrapCCCCCCCCSSSSSSde & $\begin{array}{l}2177950 \\
2178000 \\
2242+57 \\
2241+22\end{array}$ & $\begin{array}{l}\mathrm{F} \\
06 \mathrm{~N} \\
04 \mathrm{~W} \\
26\end{array}$ & $\begin{array}{ll}\text { A B } & \text { E } \\
\text { F } & \end{array}$ \\
\hline
\end{tabular}


A\&G STIPULATION 2.5.3 ZONES OF RESTRICTED ACTIVITIES - KEY FISH AREAS 07/30/03 PAGE 16 of 21

\begin{tabular}{|c|c|c|c|c|c|}
\hline MP & : STREAM NAME(s) & FISH & A: JaFeMrApMaJuJlAuSeOcNoDe & : FIELD & MER \\
\hline $\mathrm{A} / \mathrm{S}$ & Comments & : SPECIES & $\begin{array}{l}\text { : : PERIOD OF SENSITIVITY } \\
\text { A : }\end{array}$ & $\begin{array}{l}: \text { STAT1ON } \\
: \quad \text { G-5 }\end{array}$ & \\
\hline
\end{tabular}

\begin{tabular}{|c|c|c|c|c|c|c|}
\hline 413.61 & $\begin{array}{l}\text { (Tributary TATALINA RIVER) } \\
\text { TAPS A/G; LWC }\end{array}$ & $\overline{G R}$ ? & JafemrapSSSSSSauseocnode & $\begin{array}{l}2183830 \\
2183890 \\
2166+87\end{array}$ & $\begin{array}{l}\mathrm{F} \\
06 \mathrm{~N} \\
04 \mathrm{~W} \\
21\end{array}$ & $\begin{array}{l}\text { A B } \quad \text { E } \\
\text { F }\end{array}$ \\
\hline $\begin{array}{c}417.41 \\
66\end{array}$ & $\begin{array}{l}\text { GLOBE CREEK } \\
\text { TAPS A/G; BRIDGE }\end{array}$ & GR & jafemrapSSSSSSSSSSSSnode & $\begin{array}{l}2203865 \\
2203934 \\
1966+77\end{array}$ & $\begin{array}{l}\mathrm{F} \\
05 \mathrm{~N} \\
03 \mathrm{~W} \\
08\end{array}$ & $\begin{array}{l}\text { A B } \\
\text { F }\end{array}$ \\
\hline $\begin{array}{c}423.68 \\
65\end{array}$ & $\begin{array}{l}\text { North Fork AGGIE CREEK } \\
\text { TAPS A/G; LWC }\end{array}$ & GR? & JafemrapSSSSSSauseocnode & $\begin{array}{l}2236946 \\
2237126 \\
1635+99\end{array}$ & $\begin{array}{l}\mathrm{F} \\
04 \mathrm{~N} \\
03 \mathrm{~W} \\
03\end{array}$ & $\begin{array}{l}\mathrm{AB} \quad \mathrm{E} \\
\mathrm{F}\end{array}$ \\
\hline $\begin{array}{c}424.44 \\
65\end{array}$ & $\begin{array}{l}\text { South Fork AGGIE CREEK } \\
\text { TAPS A/G; LWC }\end{array}$ & GR? & JafemrapSSSSSSauseocnode & $\begin{array}{l}2241016 \\
2241077 \\
1595+22\end{array}$ & $\begin{array}{l}\mathrm{F} \\
04 \mathrm{~N} \\
03 \mathrm{~W} \\
10 \\
\end{array}$ & $\begin{array}{l}\text { A B } \quad \text { E } \\
\text { F }\end{array}$ \\
\hline $64 \mathrm{APL}-2$ & SNOWSHOE CREEK & & & & B & \\
\hline $\begin{array}{c}431.76 \\
64\end{array}$ & $\begin{array}{l}\text { WASHINGTON CREEK } \\
\text { TAPS A/G; BRIDGE }\end{array}$ & $\begin{array}{l}\text { BB?CN? } \\
\text { GR;IN? } \\
\text { NP?RW? }\end{array}$ & CCCCCCCCSSSSSSSSSSCCCCCC & $\begin{array}{l}2279644 \\
2279714 \\
1209+62\end{array}$ & $\begin{array}{l}\mathrm{F} \\
03 \mathrm{~N} \\
02 \mathrm{~W} \\
04\end{array}$ & $\begin{array}{l}\text { A B } \\
\text { F }\end{array}$ \\
\hline 62 APL-1 & VAULT CREEK & & & : & B & \\
\hline 62 APL-1 & $\begin{array}{l}\text { DOME CREEK } \\
\text { Bridge }\end{array}$ & $\begin{array}{ll}\mathrm{CN} & \\
\text { YES }\end{array}$ & & & & \\
\hline $\begin{array}{c}442.18 \\
62\end{array}$ & $\begin{array}{l}\text { TREASURE CREEK } \\
\text { TAPS A/G; BRIDGE }\end{array}$ & $\mathrm{CN} ; \mathrm{GR}$ & jafemrapSSSSSSSSSSSSnode & $\begin{array}{r}2334693 \\
2334762 \\
659+43\end{array}$ & $\begin{array}{l}\mathrm{F} \\
02 \mathrm{~N} \\
01 \mathrm{~W} \\
10\end{array}$ & $\begin{array}{ll}\text { A B } & \text { E } \\
\text { F }\end{array}$ \\
\hline $\begin{array}{c}471.05 \\
57\end{array}$ & $\begin{array}{l}\text { MOOSE CREEK \# } 3 \\
\text { TAPS B/G; BLOCKPOINT }\end{array}$ & $\begin{array}{l}\mathrm{BB} ; \mathrm{CN} ? \mathrm{GR} \\
\mathrm{HW} ; \mathrm{LS} ; \mathrm{NP} \\
\mathrm{RW} \text {;DS }\end{array}$ & $\begin{array}{l:l}Y & \text { CCCCCCCCSSSSSSSSSSCCCCCC } \\
E & \end{array}$ & $\begin{array}{l}2487100 \\
2487175 \\
1321+62 \\
1320+26 \\
\end{array}$ & $\begin{array}{l}\mathrm{F} \\
02 \mathrm{~S} \\
03 \mathrm{E} \\
19 \\
\end{array}$ & $\begin{array}{ll}\mathrm{AB} & \mathrm{E} \\
\mathrm{F} & \end{array}$ \\
\hline $\begin{array}{c}472.36 \\
57\end{array}$ & $\begin{array}{l}\text { MOOSE CREEK \# } 2 \\
\text { TAPS B/G; BRIDGE }\end{array}$ & $\begin{array}{l}\text { BB:CN?GR } \\
\text { HW;LS;NP } \\
\text { RW;DS }\end{array}$ & $\begin{array}{lll}Y & \text { CCCCCCCCSSSSSSSSSSSCCCCC } \\
E_{1} & \\
S & \end{array}$ & $\begin{array}{l}2494050 \\
2494100 \\
1250+46\end{array}$ & $\begin{array}{l}\mathrm{F} \\
02 \mathrm{~S} \\
03 \mathrm{E} \\
20\end{array}$ & $\begin{array}{ll}\mathrm{AB} & \mathrm{E} \\
\mathrm{F} & \end{array}$ \\
\hline $\begin{array}{c}474.57 \\
57\end{array}$ & $\begin{array}{l}\text { (Bear Lake Outlet) } \\
\text { TAPS B/G; CMP }\end{array}$ & $\begin{array}{l}\text { CN;CS;GR; } \\
\text { HW;LC;LS; } \\
\text { NP;RW }\end{array}$ & jafemrapCCCCSSSSSCCCCCnode & $\begin{array}{l}2505729 \\
1133+80\end{array}$ & $\begin{array}{l}\mathrm{F} \\
02 \mathrm{~S} \\
03 \mathrm{E} \\
27 \\
\end{array}$ & $\begin{array}{ll}\mathrm{AB} & \mathrm{E} \\
\mathrm{F}\end{array}$ \\
\hline $\begin{array}{c}474.74 \\
57\end{array}$ & $\begin{array}{l}\text { FRENCH CREEK } \\
\text { TAPS A/G; BRIDGE }\end{array}$ & $\begin{array}{l}\text { BB?CN?CS } \\
\text { GR;HW:LC } \\
\text { LS?NP;RW } \\
\text { SK?DS }\end{array}$ & $\begin{array}{c:c} & \text { JafemrSSSSSSSSSSSSSSSSsnode } \\
\mathrm{E}_{1} & \\
\mathrm{~S} & \end{array}$ & $\begin{array}{l}2506609 \\
2506679 \\
1125+18\end{array}$ & $\begin{array}{l}\mathrm{F} \\
02 \mathrm{~S} \\
03 \mathrm{E} \\
27\end{array}$ & $\begin{array}{ll}\mathrm{AB} & \mathrm{E} \\
\mathrm{F}\end{array}$ \\
\hline $\begin{array}{c}476.46 \\
56\end{array}$ & $\begin{array}{l}\text { FRENCH CREEK } \\
\text { TAPS B/G; BRIDGE }\end{array}$ & $\begin{array}{l}\text { BB?CN?CS } \\
\text { GR;HW:LC } \\
\text { LS?NP;RW; } \\
\text { SK?DS }\end{array}$ & 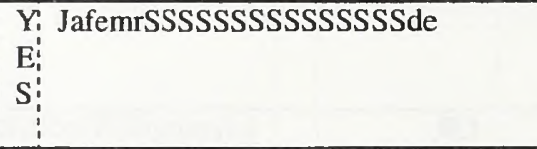 & $\begin{array}{l}2515700 \\
2515740 \\
1035+43\end{array}$ & $\begin{array}{l}\mathrm{F} \\
02 \mathrm{~S} \\
03 \mathrm{E} \\
36\end{array}$ & $\begin{array}{ll}\mathrm{AB} & \mathrm{E} \\
\mathrm{F} & \end{array}$ \\
\hline $\begin{array}{c}476.76 \\
56\end{array}$ & $\begin{array}{l}\text { FRENCH CREEK } \\
\text { TAPS B/G; BRIDGE }\end{array}$ & $\begin{array}{l}\text { BB?CN?CS? } \\
\text { GR;HW;LC } \\
\text { LS?NP;RW; } \\
\text { SK?DS }\end{array}$ & $\begin{array}{l}\mathrm{Y}: \text { JafemrSSSSSSSSSSSSSSSde } \\
\mathrm{E} \\
\mathrm{S}\end{array}$ & $\begin{array}{l}2517260 \\
2517300 \\
1018+95\end{array}$ & $\begin{array}{l}\mathrm{F} \\
03 \mathrm{~S} \\
03 \mathrm{E} \\
01\end{array}$ & $\begin{array}{ll}\mathrm{AB} & \mathrm{E} \\
\mathrm{F} & \end{array}$ \\
\hline $\begin{array}{c}477.26 \\
56\end{array}$ & $\begin{array}{l}\text { FRENCH CREEK } \\
\text { TAPS B/G; BRIDGE }\end{array}$ & $\begin{array}{l}\text { BB?CN?CS } \\
\text { GR;HW;LC } \\
\text { LS?NP;RW; } \\
\text { SK?DS }\end{array}$ & $\begin{array}{c:c}Y & \text { JafemrSSSSSSSSSSSSSSSSSde } \\
\text { E } & \\
S & \end{array}$ & $\begin{array}{r}2519900 \\
2519950 \\
993+69\end{array}$ & $\begin{array}{l}\mathrm{F} \\
035 \\
03 \mathrm{E} \\
01\end{array}$ & $\begin{array}{ll}\mathrm{AB} & \mathrm{E} \\
\mathrm{F} & \end{array}$ \\
\hline
\end{tabular}


A\&G STIPULATION 2.5.3 ZONES OF RESTRICTED ACTIVITIES - KEY FISH AREAS 07/30/03 PAGE 17 of 21

\begin{tabular}{|c|c|c|c|c|c|c|}
\hline MP & STREAM NAME(s) & $\begin{array}{l}: \text { FISH } \\
: \text { SPECIES }\end{array}$ & $\begin{array}{l}\text { : A: JaFeMrApMaJuJlAuSeOcNoDe } \\
\text { D : PERIOD OF SENSITIVITY }\end{array}$ & $\begin{array}{l}: \text { FIELD } \\
: \text { STATION }\end{array}$ & $\begin{array}{l}: \text { MER } \\
: T\end{array}$ & $\begin{array}{l}\text { : REFER } \\
: \text {-ENCE }\end{array}$ \\
\hline $\mathrm{A} / \mathrm{S}$ & : Comments & : & $\begin{array}{l}: A: \\
: D\end{array}$ & : G-5 & $\begin{array}{l}: \mathrm{R} \\
: \mathrm{SEC}\end{array}$ & : \\
\hline
\end{tabular}

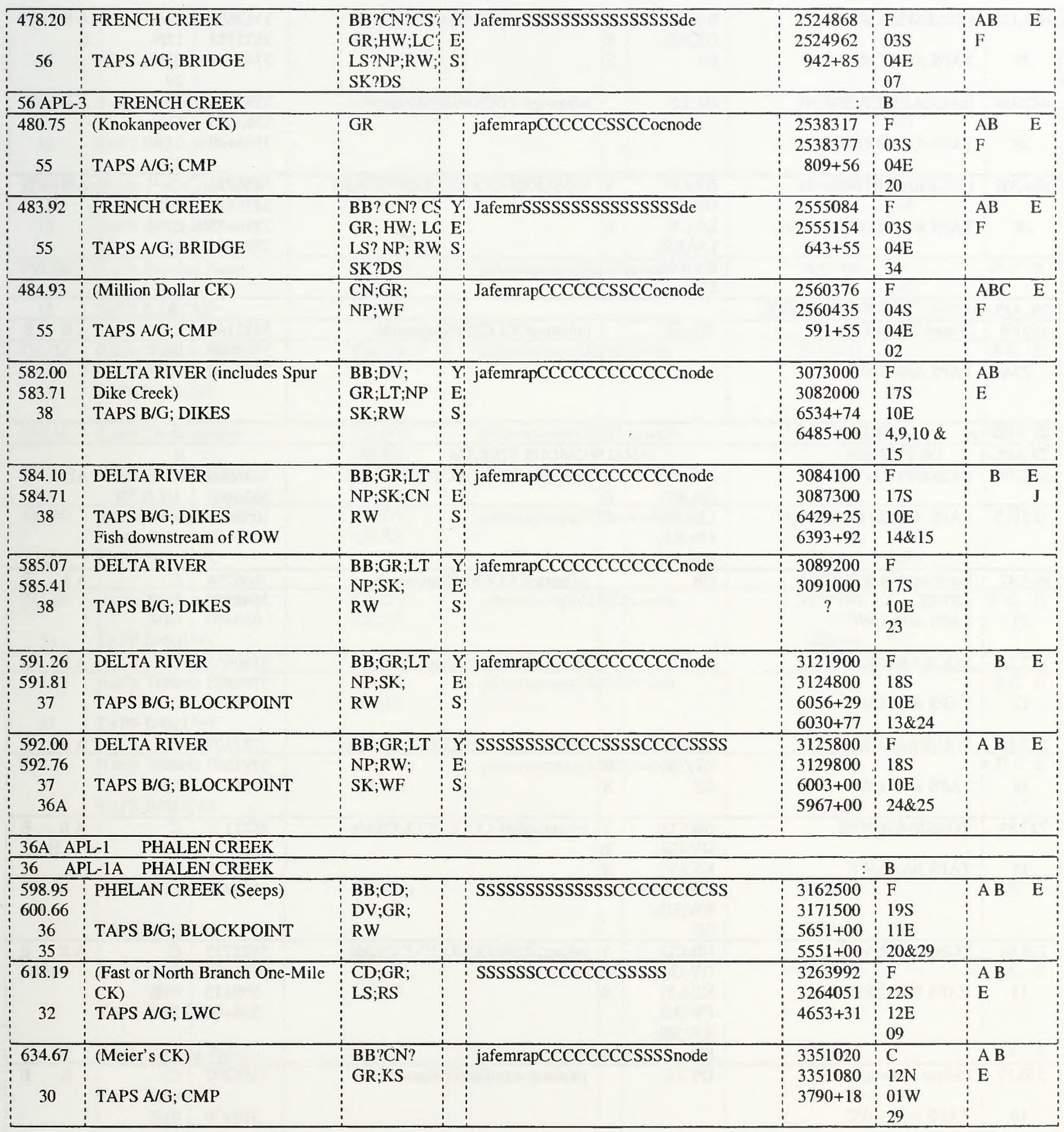


A\&G STIPULATION 2.5.3 ZONES OF RESTRICTED ACTIVITIES - KEY FISH AREAS 07/30/03 PAGE I8 of 21

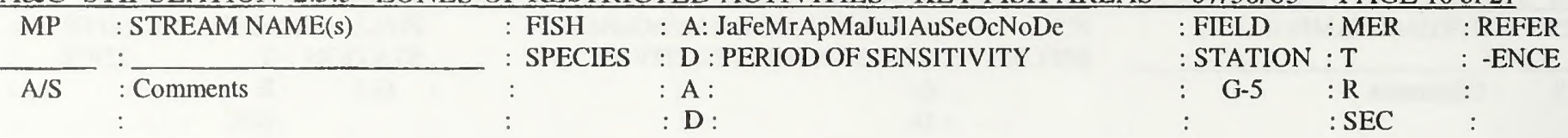

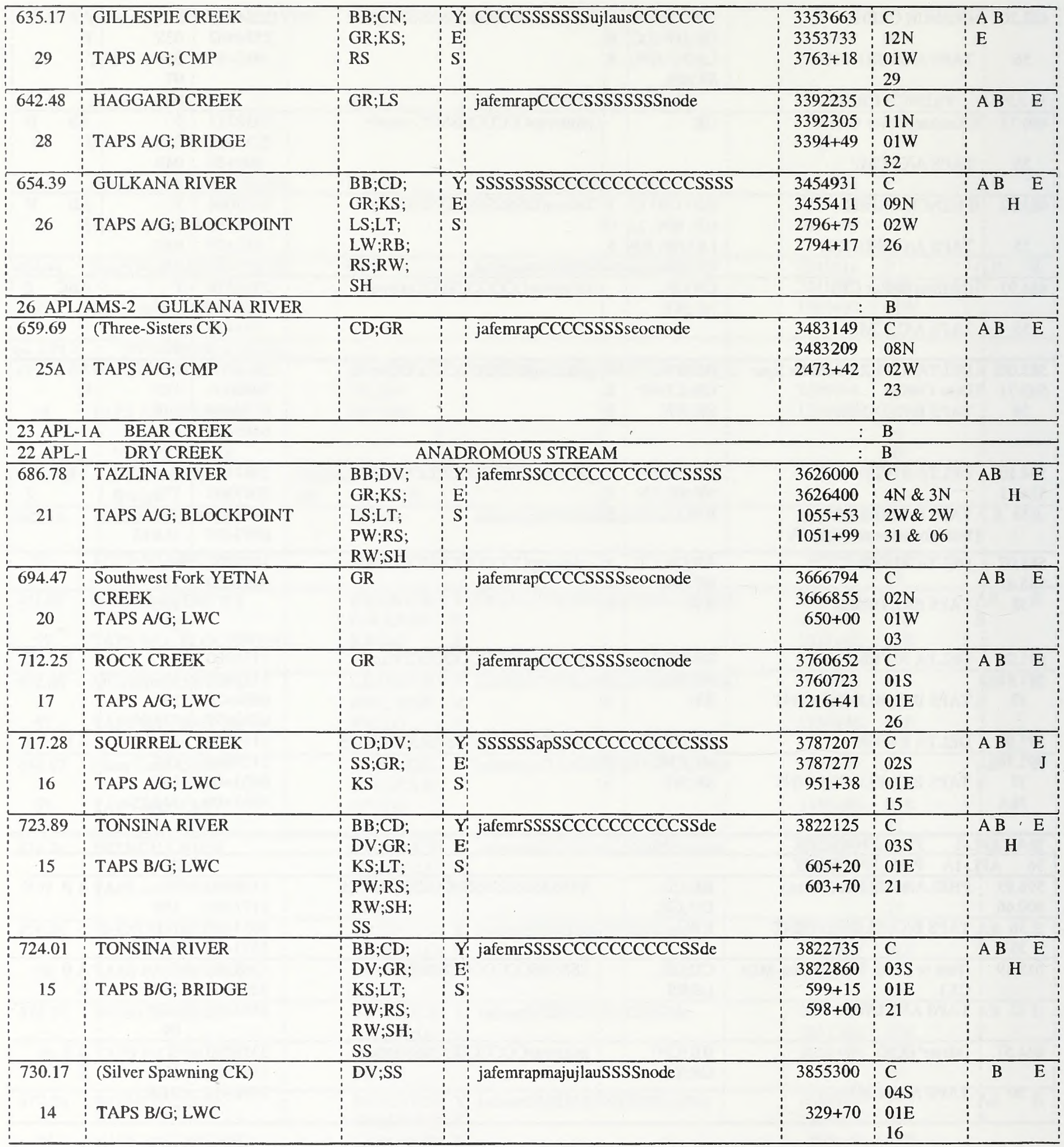


A\&G STIPULATION 2.5.3 ZONES OF RESTRICTED ACTIVITIES - KEY FISH AREAS 07/30/03 PAGE 19 of 21

\begin{tabular}{|c|c|c|c|c|c|c|}
\hline MP & : STREAM NAME(s) & : FISH & : A: JaFeMrApMaJuJlAuSeOcNoDe & : FIELD & : MER & : REFER \\
\hline & & SPECIES & : D : PERIOD OF SENSITIVITY & : STATION & $\mathrm{T}$ & : -ENCE \\
\hline A/S & $\begin{array}{l}\text { : Comments } \\
\text { : }\end{array}$ & $\begin{array}{l}: \\
:\end{array}$ & $\begin{array}{l}: \mathrm{A}: \\
: \mathrm{D}:\end{array}$ & G-5 & $\begin{array}{l}: \mathrm{R} \\
\text { SEC }\end{array}$ & : \\
\hline
\end{tabular}

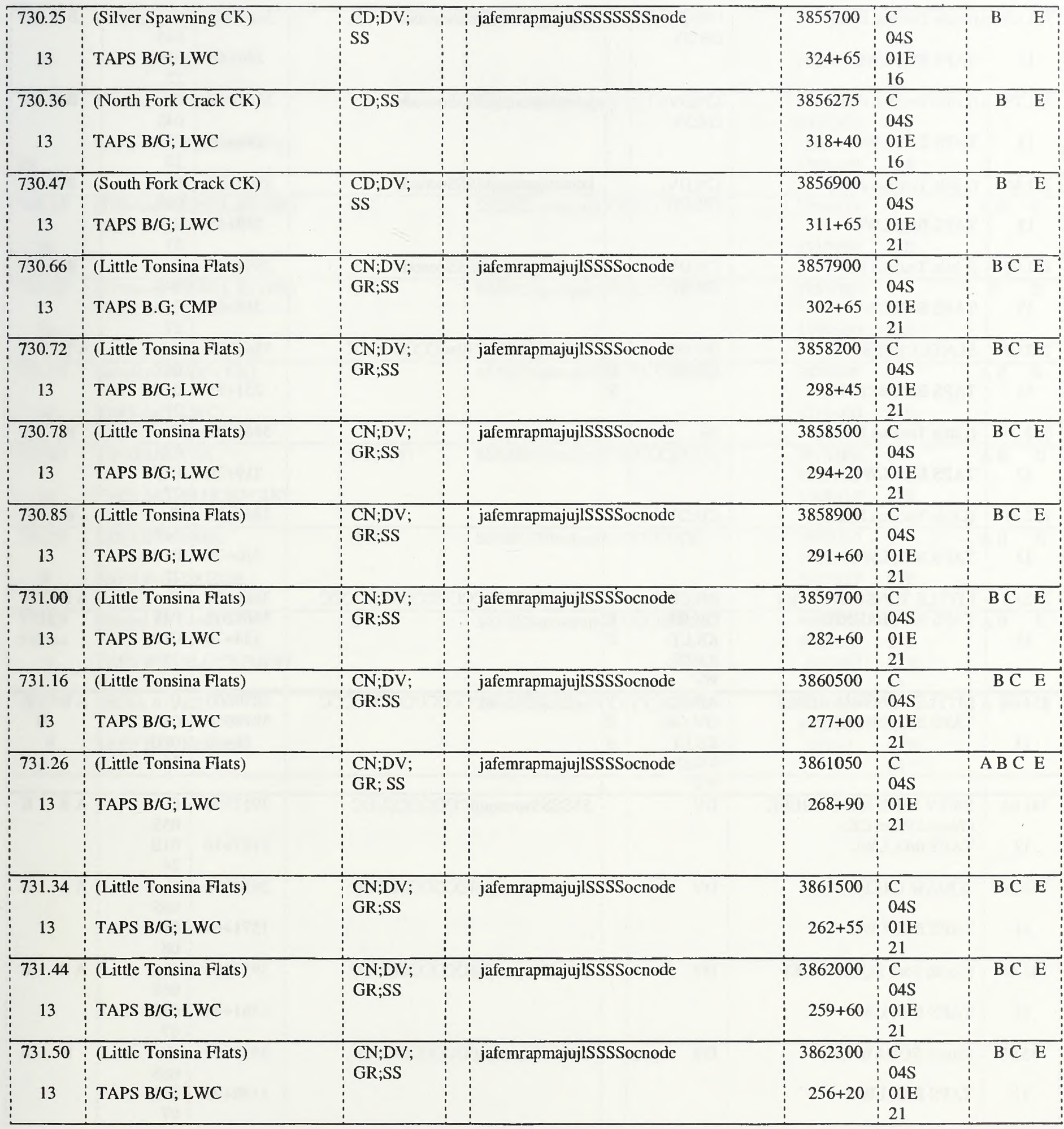


A\&G STIPULATION 2.5.3 ZONES OF RESTRICTED ACTIVITIES - KEY FISH AREAS 07/30/03 PAGE 20 of 21

\begin{tabular}{|c|c|c|c|c|c|}
\hline MP & : STREAM NAME(s) & : F1SH & : A: JaFeMrApMaJuJlAuSeOcNoDe & : FIELD & MER \\
\hline $\mathrm{A} / \mathrm{S}$ & Com & & : A : & G-5 & $: \mathrm{R}$ \\
\hline
\end{tabular}

\begin{tabular}{|c|c|c|c|c|c|c|}
\hline $\begin{array}{c}731.69 \\
13\end{array}$ & $\begin{array}{l}\text { (Little Tonsina Flats) } \\
\text { TAPS B/G; LWC }\end{array}$ & $\begin{array}{l}\text { CN;DV; } \\
\text { GR;SS }\end{array}$ & jafemrapmajujlSSSSocnode & $\begin{array}{r}3863300 \\
246+60\end{array}$ & $\begin{array}{l}\mathrm{C} \\
04 \mathrm{~S} \\
01 \mathrm{E} \\
22\end{array}$ & B C E \\
\hline $\begin{array}{c}731.78 \\
13\end{array}$ & $\begin{array}{l}\text { (Little Tonsina Flats) } \\
\text { TAPS B/G; LWC }\end{array}$ & $\begin{array}{l}\text { CN;DV; } \\
\text { GR;SS }\end{array}$ & jafemrapmajujlSSSSocnode & $\begin{array}{r}3863800 \\
244+60\end{array}$ & $\begin{array}{l}\mathrm{C} \\
04 \mathrm{~S} \\
01 \mathrm{E} \\
22\end{array}$ & B C E \\
\hline $\begin{array}{c}731.86 \\
13\end{array}$ & $\begin{array}{l}\text { (Little Tonsina Flats) } \\
\text { TAPS B/G; LWC }\end{array}$ & $\begin{array}{l}\text { CN;DV; } \\
\text { GR;SS }\end{array}$ & jafemrapmajujlSSSSocnode & $\begin{array}{r}3864200 \\
239+85\end{array}$ & $\begin{array}{l}\mathrm{C} \\
04 \mathrm{~S} \\
01 \mathrm{E} \\
27\end{array}$ & B C E \\
\hline $\begin{array}{c}731.95 \\
13\end{array}$ & $\begin{array}{l}\text { (Little Tonsina Flats) } \\
\text { TAPS B/G; LWC }\end{array}$ & $\begin{array}{l}\text { CN;DV; } \\
\text { GR;SS }\end{array}$ & jafemrapmajujlSSSSocnode & $\begin{array}{r}3864700 \\
235+65\end{array}$ & $\begin{array}{l}\mathrm{C} \\
04 \mathrm{~S} \\
01 \mathrm{E} \\
27 \\
\end{array}$ & B C E \\
\hline $\begin{array}{c}732.02 \\
13\end{array}$ & $\begin{array}{l}\text { SLATE CREEK } \\
\text { TAPS B/G; BRIDGE }\end{array}$ & $\begin{array}{l}\text { DV;GR; } \\
\text { KS;SS }\end{array}$ & $\begin{array}{c:cc}Y_{1} & \text { CCCSSSSSSSSjlauCCCCCCCC } \\
E_{1} & \\
S & \end{array}$ & $\begin{array}{r}3865050 \\
231+95\end{array}$ & $\begin{array}{l}\mathrm{C} \\
04 \mathrm{~S} \\
01 \mathrm{E} \\
27 \\
\end{array}$ & $\mathrm{ABCE}$ \\
\hline $\begin{array}{c}732.29 \\
13\end{array}$ & $\begin{array}{l}\text { (Little Tonsina Marsh) } \\
\text { TAPS B/G; LWC }\end{array}$ & SS & & $\begin{array}{r}3866500 \\
219+70\end{array}$ & $\begin{array}{l}\mathrm{C} \\
04 \mathrm{~S} \\
01 \mathrm{E} \\
27\end{array}$ & B \\
\hline $\begin{array}{c}732.41 \\
13\end{array}$ & $\begin{array}{l}\text { (Little Tonsina Marsh) } \\
\text { TAPS B/G; LWC }\end{array}$ & $\mathrm{CD} ; \mathrm{DV}$ & & $\begin{array}{r}3867100 \\
210+75\end{array}$ & $\begin{array}{l}\mathrm{C} \\
04 \mathrm{~S} \\
01 \mathrm{E} \\
27\end{array}$ & $\mathrm{~B}$ \\
\hline $\begin{array}{c}732.80 \\
13\end{array}$ & $\begin{array}{l}\text { LITTLE TONSINA RIVER } \\
\text { TAPS B/G; BRIDGE }\end{array}$ & $\begin{array}{l}\text { BB;CD; } \\
\text { DV;GR; } \\
\text { KS;LT; } \\
\text { RS;SS; } \\
\text { WF }\end{array}$ & 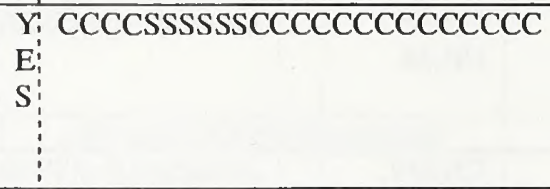 & $\begin{array}{r}3869150 \\
3869200 \\
184+74\end{array}$ & $\begin{array}{l}\mathrm{C} \\
04 \mathrm{~S} \\
01 \mathrm{E} \\
27\end{array}$ & $\underset{H}{\mathrm{ABCE}}$ \\
\hline $\begin{array}{c}734.66 \\
13\end{array}$ & $\begin{array}{l}\text { LITTLE TONSINA RIVER } \\
\text { TAPS B/G; BRIDGE }\end{array}$ & $\begin{array}{l}\mathrm{BB} ; \mathrm{CD} \\
\text { DV;GR; } \\
\mathrm{KS} ; \mathrm{LT} ; \\
\mathrm{RS} ; \mathrm{SS} ; \\
\text { WF }\end{array}$ & $\begin{array}{l}\text { Y: CCCCSSSSSSCCCCCCCCCCCCCC } \\
E_{1} \\
S:\end{array}$ & $\begin{array}{r}3879000 \\
3879050 \\
86+56\end{array}$ & $\begin{array}{l}\mathrm{C} \\
04 \mathrm{~S} \\
01 \mathrm{E} \\
36\end{array}$ & $\begin{array}{c}\text { A B C E } \\
\mathrm{H}\end{array}$ \\
\hline $\begin{array}{c}741.04 \\
12\end{array}$ & $\begin{array}{l}\text { FIFTY-NINE MILE CREEK } \\
\text { (Wagon Point CK) } \\
\text { TAPS B/G; LWC }\end{array}$ & DV & SSSSSSapmajujlCCCCCCCCCC & $\begin{array}{l}3912700 \\
1797+16\end{array}$ & $\begin{array}{l}\mathrm{C} \\
05 \mathrm{~S} \\
01 \mathrm{E} \\
26\end{array}$ & A B \\
\hline $\begin{array}{c}745.26 \\
11\end{array}$ & $\begin{array}{l}\text { SQUAW CREEK } \\
\text { TAPS B/G; LWC }\end{array}$ & DV & SSSSSSapmajujlCCCCCCCCCC & $\begin{array}{r}3934950 \\
1571+60\end{array}$ & $\begin{array}{l}\mathrm{C} \\
06 \mathrm{~S} \\
01 \mathrm{E} \\
08\end{array}$ & A B \\
\hline $\begin{array}{c}745.68 \\
11\end{array}$ & $\begin{array}{l}\text { (South Fork SQUAW CK) } \\
\text { TAPS B/G; LWC }\end{array}$ & DV & SSSSSSapmajujlCCCCCCCCCC & $\begin{array}{r}3937200 \\
1561+44\end{array}$ & $\begin{array}{l}\mathrm{C} \\
06 \mathrm{~S} \\
01 \mathrm{E} \\
07\end{array}$ & A B \\
\hline $\begin{array}{c}745.98 \\
11\end{array}$ & $\begin{array}{l}\text { (South SQUAW CK) } \\
\text { TAPS B/G; LWC }\end{array}$ & DV & SSSSSSapmajujlCCCCCCCCCC & $\begin{array}{l}3938800 \\
1548+25\end{array}$ & $\begin{array}{l}\mathrm{C} \\
06 \mathrm{~S} \\
01 \mathrm{E} \\
07\end{array}$ & B E \\
\hline
\end{tabular}




\begin{tabular}{|c|c|c|c|c|c|c|}
\hline MP & [IPULATION 2 & FISH & 1ED & $0 / 130 / 03$ & 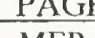 & \\
\hline & : STREAM NAME(s) & : SPECIES & D : PERIOD OF SENSITIVITY & STATION & $\mathrm{T}$ & $\begin{array}{l}\text { REFER } \\
\text {-FNCF }\end{array}$ \\
\hline $\mathrm{A} / \mathrm{S}$ & : Comn & : & $\begin{array}{l}: \mathrm{A}: \\
: \mathrm{D}:\end{array}$ & G-5 & $\begin{array}{l}: \mathrm{R} \\
: \mathrm{SEC}\end{array}$ & \\
\hline
\end{tabular}

\begin{tabular}{|c|c|c|c|c|c|c|c|}
\hline $\begin{array}{c}748.07 \\
10\end{array}$ & $\begin{array}{l}\text { BOULDER CREEK } \\
\text { TAPS B/G; LWC }\end{array}$ & DV & SSSSSSapmajujlCCCCCCCCCC & $\begin{array}{l}3949800 \\
1423+00\end{array}$ & $\begin{array}{l}\text { C } \\
06 \mathrm{~S} \\
01 \mathrm{E} \\
24\end{array}$ & $\mathrm{AB}$ & $\mathrm{E}$ \\
\hline $\begin{array}{l}749.07 \\
10\end{array}$ & $\begin{array}{l}\text { TIEKEL RIVER } \\
\text { TAPS B/G; BLOCKPOINT }\end{array}$ & $\mathrm{CD} ; \mathrm{DV}$ & SSSSSSSSmajujlCCCCCCCCCC & $\begin{array}{l}3955000 \\
3955200 \\
1369+02 \\
1368+49\end{array}$ & $\begin{array}{l}\mathrm{C} \\
06 \mathrm{~S} \\
0 \mathrm{IE} \\
25 \& \\
30\end{array}$ & $\mathrm{AB}$ & $E$ \\
\hline $\begin{array}{c}749.37 \\
10\end{array}$ & $\begin{array}{l}\text { (Tributary TIEKEL RIVER) } \\
\text { TAPS B/G; LWC }\end{array}$ & DV & SSSSSSapmajujlCCCCCCCCCC & $\begin{array}{l}3956650 \\
1357+00\end{array}$ & $\begin{array}{l}\mathrm{C} \\
06 \mathrm{~S} \\
01 \mathrm{E} \\
30\end{array}$ & $\mathrm{AB}$ & E \\
\hline $\begin{array}{c}749.43 \\
10\end{array}$ & $\begin{array}{l}\text { (Tributary TIEKEL RIVER) } \\
\text { TAPS B/G; CMP }\end{array}$ & DV & SSSSSSapmajujlCCCCCCCCCC & $\begin{array}{l}3957000 \\
1349+80\end{array}$ & $\begin{array}{l}\mathrm{C} \\
06 \mathrm{~S} \\
01 \mathrm{E} \\
30\end{array}$ & $\mathrm{~B}$ & $\mathrm{E}$ \\
\hline $\begin{array}{c}751.95 \\
10\end{array}$ & $\begin{array}{l}\text { (Small or Jackic's CK) } \\
\text { TAPS B/G; LWC }\end{array}$ & DV & SSSSSSapmajujlCCCCCCCCCC & $\begin{array}{l}3970300 \\
\text { I } 216+00\end{array}$ & $\begin{array}{l}\mathrm{C} \\
07 \mathrm{~S} \\
01 \mathrm{E} \\
05\end{array}$ & A B & $\mathrm{E}$ \\
\hline $\begin{array}{c}752.87 \\
10\end{array}$ & $\begin{array}{l}\text { TIEKEL RIVER } \\
\text { TAPS B/G; BLOCKPOINT }\end{array}$ & $\mathrm{CD} ; \mathrm{DV}$ & SSSSSSSSmajujlCCCCCCCCCC & $\begin{array}{l}3975100 \\
3975200 \\
\text { I I } 68+18 \\
1167+45\end{array}$ & $\begin{array}{l}\mathrm{C} \\
07 \mathrm{~S} \\
0 \mathrm{IE} \\
08\end{array}$ & A B & $\mathrm{E}$ \\
\hline $\begin{array}{c}754.19 \\
9\end{array}$ & $\begin{array}{l}\text { STUART CREEK } \\
\text { TAPS B/G; BRIDGE }\end{array}$ & DV & SSSSSSSSmajujlaCCCCCCCC & $\begin{array}{l}3982000 \\
3982200 \\
1097+77\end{array}$ & $\begin{array}{l}\mathrm{C} \\
07 \mathrm{~S} \\
01 \mathrm{E} \\
18 \\
\end{array}$ & A B & $E$ \\
\hline $\begin{array}{c}758.29 \\
758.64 \\
9\end{array}$ & $\begin{array}{l}\text { (Gravel Pit Pond) } \\
\text { TAPS B/G; BLOCKPOINT }\end{array}$ & $\mathrm{DV}$ & SSSSSSapmajujlCCCCCCCCCC & $\begin{array}{r}4003750 \\
4005640 \\
868+87 \\
863+65 \\
\end{array}$ & $\begin{array}{l}\mathrm{C} \\
07 \mathrm{~S} \\
0 \mathrm{IW} \\
34 \\
\end{array}$ & A B & $\mathrm{E}$ \\
\hline $\begin{array}{c}759.05 \\
9\end{array}$ & $\begin{array}{l}\text { TSINA RIVER } \\
\text { TAPS B/G; BRIDGE }\end{array}$ & DV & SSSSSSapmajujlCCCCCCCCCC & $\begin{array}{r}4007650 \\
4007900 \\
842+38\end{array}$ & $\begin{array}{l}\mathrm{C} \\
07 \mathrm{~S} \\
01 \mathrm{~W} \\
34\end{array}$ & $\mathrm{AB}$ & $\bar{E}$ \\
\hline
\end{tabular}




\section{Selected References}

(A)

Alaska Pipeline Office. 1977. Interim report on zones of restricted activity for fish and wildlife along the Trans-Alaska Pipeline. U.S. Dept. of the Interior. Anchorage, Alaska. (February 16, 1977; 37pp).

(B)

Johnson, Richard L. and Julius Rockwell, Jr. (Revised by J. Rockwell, Jr.). 1981. List of streams and other water bodies along the Trans-Alaska oil pipeline route (Fourth Revision: Draft). U.S. Dept. of the Interior, Alaska Pipeline Office, Anchorage, Alaska (May 1, 1981).

(C)

Elliott, George V. 1980. First interim report on the evaluation of stream crossings and effects of channel modifications on fishery resources along the route of the Trans-Alaska Pipeline. U.S. Fish and Wildlife Service, Anchorage, Alaska (June, 1980: 77pp).

(D)

Elliott, George V. 1982. Final report on the evaluation of stream crossings and effects of channel modifications on fishery resources along the route of the Trans-Alaska Pipeline. U.S. Fish and Wildlife Service, Anchorage, Alaska (March 1982;) 110 pp).

(E)

Office of Special Projects. 1982. [no title]. U.S. Bureau of Land Management, Anchorage, Alaska. (May 6, 1982). [This list is commonly referred to as Ken Hunt's list and includes comments from the Alaska Dept. of Fish and Game].

(F)

Office of the Federal Inspector for ANGTS. 1984. List of fish stream data. Anchorage, Alaska (February 13,$1984 ; 50 \mathrm{pp})$.

(G)

DenBeste, J. and P. McCart. 1984. Catalog of streams associated with the Trans Alaska pipeline System in the northern district. Volume IV. Prepared for Alyeska Pipeline Service Company by Aquatic Environments Inc., Anchorage, Alaska. (April 1984; 67 pp).

(H)

Roberson, Kenneth. 1985 (letter of 12/18). [Comments on First Edition of 1/1/86]. Alaska Dept. of Fish and Game, Glenallen, Alaska.

(I)

Alyeska Pipeline Service Co. 1986 (letter of May 12, No. 86-3642) [Comments on First Edition of 1/1/ 86]. Anchorage, Alaska.

Anadromous Fishes

Alaska Department of Fish and Game, Habitat Division. 1985. Catalog of waters important for spawning, rearing or migration of anadromous fishes, as revised March 29, 1985 effective May 19, 1985; Regions II, V, and VI. Juneau, Alaska.

(J)

Gnath, D.G., D.W. Lieb, and M. Wiedmer. 2002. Trans-Alaska Pipeline System 2002 Fish Habitat Survey.Alaska Department of Fish and Game, Habitat and Restoration Division, Technical Report No. 02-07, Anchorage, Alaska. 


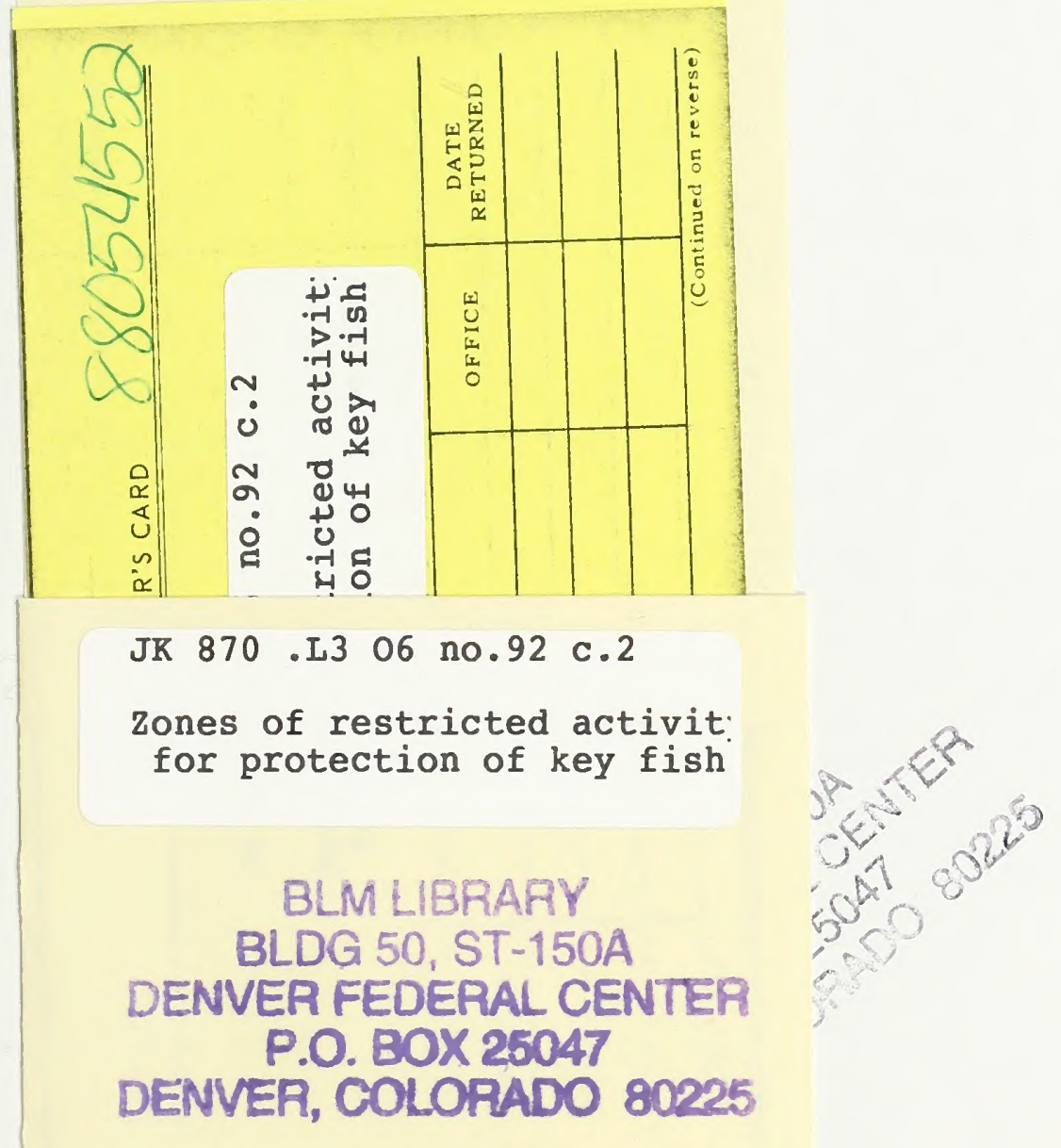


